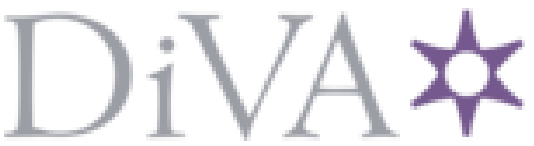

http://www.diva-portal.org

Preprint

This is the submitted version of a paper published in Journal of Computational and Applied Mathematics.

Citation for the original published paper (version of record):

Lin, G., Cheng, X., Zhang, Y. (2018)

A parametric level set based collage method for an inverse problem in elliptic partial differential equations.

Journal of Computational and Applied Mathematics

Access to the published version may require subscription.

N.B. When citing this work, cite the original published paper.

Permanent link to this version:

http://urn.kb.se/resolve?urn=urn:nbn:se:oru:diva-64605 
Elsevier Editorial System(tm) for Journal of Computational and Applied Mathematics

Manuscript Draft

Manuscript Number: CAM-D-16-02024R3

Title: A parametric level set based collage method for an inverse problem in elliptic partial differential equations

Article Type: Research Paper

Section/Category: 47A52 (Ill-posed problems, regularization)

Keywords: Inverse problem; Collage theorem; Regularization; Parametric level set; Total variation.

Corresponding Author: Dr. Ye Zhang, Ph.D.

Corresponding Author's Institution: School of Science and Technology

First Author: Guangliang Lin

Order of Authors: Guangliang Lin; Xiaoliang Cheng; Ye Zhang, Ph.D.

Abstract: In this work, based on the collage theorem, we develop a new numerical approach to reconstruct the locations of discontinuity of the conduction coefficient in elliptic partial differential equations (PDES) with inaccurate measurement data and coefficient value. For a given conductivity coefficient, one can construct a contraction mapping such that its fixed point is just the gradient of a solution to the elliptic system. Therefore, the problem of reconstructing a conductivity coefficient in PDEs can be considered as an approximation of the observation data by the fixed point of a contraction mapping. By collage theorem, we translate it to seek a contraction mapping that keeps the observation data as close as possible to itself, which avoids solving adjoint problems when applying the gradient descent method to the corresponding optimization problem. Moreover, the total variation regularizing strategy is applied to tackle the ill-posedness and the parametric level set technique is adopted to represent the discontinuity of the conductivity coefficient. Various numerical simulations are given to show the efficiency of the proposed method. 


\title{
A parametric level set based collage method for an inverse problem in elliptic partial differential equations
}

\author{
Guangliang Lin ${ }^{\mathrm{a}}$, Xiaoliang Cheng ${ }^{\mathrm{a}}$, Ye Zhang \\ ${ }^{a}$ School of Mathematics, Zhejiang University, Hangzhou, Zhejiang, 310027, China \\ ${ }^{b}$ School of Science and Technology, Örebro University, Örebro, SE-701 82, Sweden \\ ${ }^{c}$ Faculty of Mathematics, Technische Universität Chemnitz, D-09107 Chemnitz, Germany
}

\begin{abstract}
In this work, based on the collage theorem, we develop a new numerical approach to reconstruct the locations of discontinuity of the conduction coefficient in elliptic partial differential equations (PDEs) with inaccurate measurement data and coefficient value. For a given conductivity coefficient, one can construct a contraction mapping such that its fixed point is just the gradient of a solution to the elliptic system. Therefore, the problem of reconstructing a conductivity coefficient in PDEs can be considered as an approximation of the observation data by the fixed point of a contraction mapping. By collage theorem, we translate it to seek a contraction mapping that keeps the observation data as close as possible to itself, which avoids solving adjoint problems when applying the gradient descent method to the corresponding optimization problem. Moreover, the total variation regularizing strategy is applied to tackle the ill-posedness and the parametric level set technique is adopted to represent the discontinuity of the conductivity coefficient. Various numerical simulations are given to show the efficiency of the proposed method.
\end{abstract}

Keywords: Inverse problem, Partial differential equations, Collage theorem, Regularization, Parametric level set, Total variation

\section{Introduction}

We are interested in reconstructing the locations of discontinuity of the conduction coefficient $\sigma(\mathbf{x}) \in L^{\infty}(\Omega)$ in the following elliptic differential equation

$$
\left\{\begin{aligned}
-\nabla \cdot(\sigma \nabla u) & =f & & \text { in } \Omega, \\
u & =0 & & \text { on } \partial \Omega,
\end{aligned}\right.
$$

where $\Omega$ is a bounded closed domain in $\mathbb{R}^{N}(N \in\{1,2,3\})$ with piecewise smooth boundary $\partial \Omega$ and the source $f \in H^{-1}(\Omega)$ is given. The applications of the above inverse problem in the elliptic system can be found in many industrial problems, such as geophysical problems [1, 2], medical imaging [3], water resource problems [4], etc. If the coefficient value is unknown, some effective methods have been employed to find

\footnotetext{
${ }^{*}$ Corresponding author.

Email addresses: guanglianglin@zju.edu.cn (Guangliang Lin), xiaoliangcheng@zju.edu.cn (Xiaoliang Cheng), ye.zhang@oru.se (Ye Zhang)
} 
a part of information of $\sigma(\mathbf{x})$, including the locations of its discontinuity, see $[5,6]$ and references therein. Recently, the level set method has been extended for this kind of problem $[7,8,9,10,11,12,13]$. In [11], the level set method with total variation regularization has been applied to this problem. In [12], the author adopted the piecewise constant level set method. The parametrization of the level set function has motivated some authors to use it in many applications $[14,15,16,17,18,19]$. The main idea of this paper is to combine the parametric level set method and the collage method to develop an efficient numerical algorithm for reconstructing the locations of discontinuity of the coefficient $\sigma$ in the boundary value problem (BVP) (1). We highlight the essential contributions of our work in the following:

- We formulate the collage method in a variational setting. One of the advantages of this formulation is to avoid solving adjoint problems when applying gradient descent methods to the corresponding optimization problem. Moreover, instead of dealing with a weighted data fitting problem for traditional approaches, the collage method works on the equivalent problem to the original data fitting problem, which is more reasonable from a practical point of view.

- We apply a parametric level set method for BVP (1). As the result of the parametrization, it's easy to obtain the regularization norm, which corresponds to the total boundary length of the recovered shapes. Furthermore, the parametric level set method greatly reduces the computational cost and overcomes some difficulties with the traditional level set method such as reinitialization, narrow-banding, etc.

- Based on the discrepancy principle, we develop a regularization parameter selection method for the identification problem of the locations of discontinuity of the coefficient in BVP (1). As far as we are aware, this is the first time that a regularization parameter selection method has been taken into account for the reconstruction of the locations of discontinuity of the coefficient in PDEs.

Now, let us formulate the inverse problem. Suppose that we a priori know a part of the information regarding the unknown exact coefficient $\bar{\sigma}$ :

$$
\bar{\sigma}=\bar{\sigma}(\mathbf{x})= \begin{cases}\bar{\sigma}_{1}(\mathbf{x}) & \text { when } \mathbf{x} \in D \subset \Omega \\ \bar{\sigma}_{2}(\mathbf{x}) & \text { when } \mathbf{x} \in \Omega \backslash D\end{cases}
$$

However, the geometry structure of domain $D$ is unknown. Assume that $\bar{\sigma}, \bar{\sigma}_{i} \in$ $L^{\infty}(\Omega)$ and instead of exact data $\left\{\bar{\sigma}_{1}, \bar{\sigma}_{2}\right\}$ we are given approximate data $\left\{\sigma_{1}^{\varepsilon}, \sigma_{2}^{\varepsilon}\right\}$ $\left(\sigma_{i}^{\varepsilon} \in L^{\infty}(\Omega)\right)$, such that

$$
\left\|\sigma_{i}^{\varepsilon}-\bar{\sigma}_{i}\right\|_{\infty} \leq \varepsilon, \quad i=1,2,
$$

where $\|\sigma\|_{\infty}:=\max _{\mathbf{x} \in \Omega}|\sigma(\mathbf{x})|$. Denote $\bar{u}$ as the solution to the BVP (1) with the exact unknown coefficient $\bar{\sigma}$. Denote $L^{2}(\Omega)^{N} \equiv L^{2}(\Omega) \times \cdots \times L^{2}(\Omega)$ and let observation data ${ }^{\epsilon} \nabla u$ satisfy

$$
\left\|{ }^{\epsilon} \nabla u-\nabla \bar{u}\right\|_{L^{2}(\Omega)^{N}} \leq \epsilon,
$$

where norm $\|\bullet\|_{L^{2}(\Omega)^{N}}$ is associated with the inner product $\langle f, g\rangle_{L^{2}(\Omega)^{N}}\left(\equiv \int_{\Omega} f \cdot g d \mathbf{x}\right)$, and the operation "." is defined as $f \cdot g=\sum_{i=1}^{N} f_{i} \cdot g_{i}$ for all $f, g \in L^{2}(\Omega)^{N}$. Here, 
constants $\varepsilon$ and $\epsilon$ represent the error levels of data, and the collection of the gradient data ${ }^{\epsilon} \nabla u$ will be discussed in Section 4. Then, the regularized output least squares (OLS) approach for the above inverse problem can be described as the following problem

$$
\min _{\sigma} \frac{1}{2}\left\|\nabla u(\sigma)-{ }^{\epsilon} \nabla u\right\|_{L^{2}(\Omega)^{N}}^{2}+\alpha \Psi(\sigma),
$$

where $u(\sigma)$ solves BVP (1) with a fixed coefficient $\sigma$. Here $\alpha=\alpha(\varepsilon, \epsilon)>0$ is the socalled regularization parameter and the second term in the cost functional represents a regularization term, which guarantees the continuity of the mappings from the observation data to the solution for the appropriate choice of $\Psi(\cdot)$ and $\alpha$.

However, the formulation (5) cannot be solved by gradient-based algorithms due to the difficult calculation of the gradient of functional $\left\|\nabla u(\sigma)-{ }^{\epsilon} \nabla u\right\|_{L^{2}(\Omega)^{N}}^{2}$. Alternatively, researchers prefer to investigate the following optimization problem

$$
\min _{\sigma} \frac{1}{2} \int_{\Omega} \sigma\left(\nabla u(\sigma)-{ }^{\epsilon} \nabla u\right) \cdot\left(\nabla u(\sigma)-{ }^{\epsilon} \nabla u\right) d \mathbf{x}+\alpha \Psi(\sigma)=: \min _{\sigma} \tilde{\mathcal{J}}(\sigma),
$$

see [6] and references therein. It has to been noted that the relation between the two problems (5) and (6) is unclear in the general case. Hence, an essential contribution of our paper is to study the formulation (5) directly by the collage method.

The remainder of the paper is structured as follows. In the first part of Section 2, based on the collage theorem, we develop a new OLS approach for reconstructing the locations of discontinuity of the coefficient in BVP (1) - the collage method. Some properties of the collage method are discussed in the second part of Section 2. In Section 3, based on the proposed collage method, a parametric level set technique is incorporated to solve the problem. Methods of calculating the gradient of the objective functional and level set function are developed in the same section. Section 4 presents the approximation technique of estimating the effective gradient data by the solution data. The regularization properties of the developed collage method are discussed in Section 5. Section 6 describes the numerical implementation of the collage method. Various numerical examples are presented in Section 7 to demonstrate the robustness of the proposed method. Finally, concluding remarks are given in Section 8 .

\section{The collage method}

Broad classes of inverse problems can be cast in the following framework: the optimal approximation of observation data $\mathbf{x}_{o b s}$ in a suitable complete metric space $\left(X, \rho_{X}\right)$ by the fixed point $\overline{\mathbf{x}}$ of a contractive mapping $T: X \rightarrow X$. In practice, from a family of parametric contraction mappings $\left\{T^{\sigma}\right\}_{\sigma}$, one wishes to find the parameter $\sigma$ for which the approximation error $\rho_{X}\left(\overline{\mathbf{x}}^{\sigma}, \mathbf{x}_{\text {obs }}\right)$ is as small as possible. A simplification of this problem is supported by the following theorem [20,21].

Theorem 1. (Collage and anti-collage theorem) Let $\left(X, \rho_{X}\right)$ be a complete metric space and $T: X \rightarrow X$ be a contraction mapping with contraction factor $\lambda \in[0,1)$. Then for any $\mathbf{x} \in X$ :

$$
\frac{1}{1+\lambda} \rho_{X}(\mathbf{x}, T \mathbf{x}) \leq \rho_{X}(\mathbf{x}, \overline{\mathbf{x}}) \leq \frac{1}{1-\lambda} \rho_{X}(\mathbf{x}, T \mathbf{x})
$$

where $\overline{\mathbf{x}}$ is the fixed point of operator $T$. 
By the relation (7), one can refer to $\rho_{X}(\mathbf{x}, T \mathbf{x})$ as the collage distance in $X$. If $X$ is a Banach space with associated norm $\|\bullet\|_{X}$, then, Theorem $\mathbf{1}$ replaces the minimization of the approximation error $\left\|\overline{\mathbf{x}}^{\sigma}-\mathbf{x}_{o b s}\right\|_{X}$ by the minimization of the collage distance $\left\|T^{\sigma} \mathbf{x}_{o b s}-\mathbf{x}_{o b s}\right\|_{X}$ since one cannot find $\overline{\mathbf{x}}^{\sigma}$ for a general $T^{\sigma}$. This is the essence of collage method which has been the basis of many inverse problems and related fields $[22,21,23,24]$. In this paper, based on the Theorem 1 we proposed a collage method for reconstructing the locations of discontinuity of the coefficient in BVP (1).

\subsection{The collage method}

The variational formulation for BVP (1) is depicted as follows: find $u \in H_{0}^{1}(\Omega)$ such that

$$
\int_{\Omega} \sigma \nabla u \cdot \nabla v d \mathbf{x}=\int_{\Omega} f v d \mathbf{x}, \quad \forall v \in H_{0}^{1}(\Omega) .
$$

We construct a nonlinear contraction mapping $T^{\sigma}: L^{2}(\Omega)^{N} \rightarrow L^{2}(\Omega)^{N}$ such that for all $\nabla u \in L^{2}(\Omega)^{N}$ :

$$
\int_{\Omega} d \cdot T^{\sigma} \nabla u \cdot \nabla v d \mathbf{x}=\int_{\Omega} f v+(d-\sigma) \nabla u \cdot \nabla v d \mathbf{x}, \quad \forall v \in H_{0}^{1}(\Omega),
$$

where $d=d(\mathbf{x})>0$ is a contraction parameter which guarantees the contraction property of the mapping $T^{\sigma}$ (see Theorem 2 for details). Obviously, for any given $\sigma(\mathbf{x})$, the gradient of a solution to (8) is a fixed point of $T^{\sigma}$. By Theorem 1, the optimization problem (5) can be reformulated as the following one

$$
\min _{\sigma} \frac{1}{2}\left\|T^{\sigma \epsilon} \nabla u-{ }^{\epsilon} \nabla u\right\|_{L^{2}(\Omega)^{N}}^{2}+\alpha \Psi(\sigma)=: \min _{\sigma} \mathcal{J}(\sigma),
$$

where parametric operator $T^{\sigma}$ is defined by (9).

\subsection{Analysis of the collage method}

First, let us discuss the conditions under which $T^{\sigma}$ is a contraction mapping. Assume $d(\mathbf{x}), 1 / d(\mathbf{x}) \in L^{\infty}(\Omega)$ and define a contraction number $\lambda(d, \sigma)$ as

$$
\lambda(d, \sigma)=\left\|d^{-1}\right\|_{\infty} \cdot\left\|d^{-1} \cdot(d-\sigma)\right\|_{\infty} \cdot\|d-\sigma\|_{\infty} .
$$

Then, the following theorem holds.

Theorem 2. If the contraction number $\lambda(d, \sigma)$ in (11) is less than unit, then $T^{\sigma}$ is a contraction mapping in the Banach space $\left(L^{2}(\Omega)^{N},\|\cdot\|_{L^{2}(\Omega)^{N}}\right)$.

Proof. For fixed $d$ and $\sigma, \forall u^{1}, u^{2} \in H_{0}^{1}(\Omega)$, we have

$$
\begin{aligned}
& \int_{\Omega} d \cdot T^{\sigma} \nabla u^{1} \cdot \nabla v d \mathbf{x}=\int_{\Omega} f v+(d-\sigma) \nabla u^{1} \cdot \nabla v d \mathbf{x}, \\
& \int_{\Omega} d \cdot T^{\sigma} \nabla u^{2} \cdot \nabla v d \mathbf{x}=\int_{\Omega} f v+(d-\sigma) \nabla u^{2} \cdot \nabla v d \mathbf{x}
\end{aligned}
$$


for $\forall v \in H_{0}^{1}(\Omega)$. Subtracting (13) from (12), we obtain

$$
\int_{\Omega} d \cdot\left(T^{\sigma} \nabla u^{1}-T^{\sigma} \nabla u^{2}\right) \cdot \nabla v d \mathbf{x}=\int_{\Omega}(d-\sigma)\left(\nabla u^{1}-\nabla u^{2}\right) \cdot \nabla v d \mathbf{x}
$$

for $\forall v \in H_{0}^{1}(\Omega)$. Replace $\nabla v$ by $T^{\sigma} \nabla u^{1}-T^{\sigma} \nabla u^{2}$ and $\nabla u^{1}-\nabla u^{2}$ we obtain

$$
\begin{aligned}
& \int_{\Omega} d \cdot\left(T^{\sigma} \nabla u^{1}-T^{\sigma} \nabla u^{2}\right) \cdot\left(T^{\sigma} \nabla u^{1}-T^{\sigma} \nabla u^{2}\right) d \mathbf{x} \\
= & \int_{\Omega}(d-\sigma)\left(\nabla u^{1}-\nabla u^{2}\right) \cdot\left(T^{\sigma} \nabla u^{1}-T^{\sigma} \nabla u^{2}\right) d \mathbf{x}
\end{aligned}
$$

and

$$
\begin{aligned}
& \int_{\Omega} d \cdot\left(T^{\sigma} \nabla u^{1}-T^{\sigma} \nabla u^{2}\right) \cdot\left(\nabla u^{1}-\nabla u^{2}\right) d \mathbf{x} \\
= & \int_{\Omega}(d-\sigma)\left(\nabla u^{1}-\nabla u^{2}\right) \cdot\left(\nabla u^{1}-\nabla u^{2}\right) d \mathbf{x} .
\end{aligned}
$$

Therefore, we can conclude

$$
\begin{aligned}
& \left\|T^{\sigma} \nabla u^{1}-T^{\sigma} \nabla u^{2}\right\|_{L^{2}(\Omega)^{N}}^{2}=\int_{\Omega}\left(T^{\sigma} \nabla u^{1}-T^{\sigma} \nabla u^{2}\right) \cdot\left(T^{\sigma} \nabla u^{1}-T^{\sigma} \nabla u^{2}\right) d \mathbf{x} \\
& \leq\left\|d^{-1}\right\|_{\infty} \cdot \int_{\Omega} d \cdot\left(T^{\sigma} \nabla u^{1}-T^{\sigma} \nabla u^{2}\right) \cdot\left(T^{\sigma} \nabla u^{1}-T^{\sigma} \nabla u^{2}\right) d \mathbf{x} \quad \text { (Hölder inequality) } \\
& =\left\|d^{-1}\right\|_{\infty} \cdot \int_{\Omega}(d-\sigma)\left(T^{\sigma} \nabla u^{1}-T^{\sigma} \nabla u^{2}\right) \cdot\left(\nabla u^{1}-\nabla u^{2}\right) d \mathbf{x} \quad(\operatorname{By}(14)) \\
& \leq\left\|d^{-1}\right\|_{\infty} \cdot\left\|\frac{d-\sigma}{d}\right\|_{\infty} \cdot \int_{\Omega} d \cdot\left(T^{\sigma} \nabla u^{1}-T^{\sigma} \nabla u^{2}\right) \cdot\left(\nabla u^{1}-\nabla u^{2}\right) d \mathbf{x} \\
& =\left\|d^{-1}\right\|_{\infty} \cdot\left\|\frac{d-\sigma}{d}\right\|_{\infty} \cdot \int_{\Omega}(d-\sigma)\left(\nabla u^{1}-\nabla u^{2}\right) \cdot\left(\nabla u^{1}-\nabla u^{2}\right) d \mathbf{x} \quad(\operatorname{By}(15)) \\
& \leq\left\|d^{-1}\right\|_{\infty} \cdot\left\|\frac{d-\sigma}{d}\right\|_{\infty} \cdot\|d-\sigma\|_{\infty} \cdot \int_{\Omega}\left(\nabla u^{1}-\nabla u^{2}\right) \cdot\left(\nabla u^{1}-\nabla u^{2}\right) d \mathbf{x} \\
& =\lambda(d, \sigma) \cdot\left\|\nabla u^{1}-\nabla u^{2}\right\|_{L^{2}(\Omega)^{N}}^{2} .
\end{aligned}
$$

By the definition of contraction mapping, operator $T^{\sigma}$ defined in (9) is really a contraction mapping under the assumption $\lambda(d, \sigma)<1$.

Now, let us show a method of choosing the contraction parameter $d(\mathbf{x})$, for which the corresponding contraction number $\lambda$ is less than unit.

Lemma 3. Let $\underline{\sigma}$ be a positive number. For the conductivity coefficient with structure

$$
\sigma(\mathbf{x})=\left\{\begin{array}{l}
\sigma_{1}(\mathbf{x}) \geq \underline{\sigma}>0, \quad \mathbf{x} \in D \\
\sigma_{2}(\mathbf{x}) \geq \underline{\sigma}>0, \quad \mathbf{x} \in \Omega \backslash D
\end{array}\right.
$$

define

$$
\tilde{d}:=\frac{\left\|\sigma_{1}^{\varepsilon}\right\|_{\infty}+\left\|\sigma_{2}^{\varepsilon}\right\|_{\infty}}{2}
$$

where $\left\{\sigma_{1}^{\varepsilon}, \sigma_{2}^{\varepsilon}\right\}$ is an approximate data of $\left\{\sigma_{1}, \sigma_{2}\right\}$ such that $\left\|\sigma_{i}^{\varepsilon}-\sigma_{i}\right\|_{\infty} \leq \varepsilon, i=1,2$. Then, $\exists \varepsilon_{0}, \forall \varepsilon \in\left[0, \varepsilon_{0}\right): \lambda(\tilde{d}, \sigma)<1$. 
Proof. Without loss of generality, consider the case when $\left\|\sigma_{2}\right\|_{\infty} \geq\left\|\sigma_{1}\right\|_{\infty}$. By definition of $\sigma_{i}^{\varepsilon}$ and triangle inequality of a norm, we have the following relations

$$
\left\|\sigma_{i}\right\|_{\infty} \leq\left\|\sigma_{2}\right\|_{\infty} \leq\left\|\sigma_{2}^{\varepsilon}\right\|_{\infty}+\varepsilon
$$

which implies

$$
\begin{aligned}
& \left\|\left(\left\|\sigma_{1}^{\varepsilon}\right\|_{\infty}+\left\|\sigma_{2}^{\varepsilon}\right\|_{\infty}-2 \sigma_{i}\right)\right\|_{\infty} \\
& =\max \left\{\sup _{\mathbf{x} \in \Omega}\left\{\left\|\sigma_{1}^{\varepsilon}\right\|_{\infty}+\left\|\sigma_{2}^{\varepsilon}\right\|_{\infty}-2 \sigma_{i}\right\}, \sup _{\mathbf{x} \in \Omega}\left\{2 \sigma_{i}-\left\|\sigma_{1}^{\varepsilon}\right\|_{\infty}-\left\|\sigma_{2}^{\varepsilon}\right\|_{\infty}\right\}\right\} \\
& \leq \max \left\{\left\|\sigma_{1}^{\varepsilon}\right\|_{\infty}+\left\|\sigma_{2}^{\varepsilon}\right\|_{\infty}-2 \underline{\sigma}, 2\left\|\sigma_{i}\right\|_{\infty}-\left\|\sigma_{1}^{\varepsilon}\right\|_{\infty}-\left\|\sigma_{2}^{\varepsilon}\right\|_{\infty}\right\} \\
& \leq \max \left\{\left\|\sigma_{1}^{\varepsilon}\right\|_{\infty}+\left\|\sigma_{2}^{\varepsilon}\right\|_{\infty}-2 \underline{\sigma},\left\|\sigma_{2}^{\varepsilon}\right\|_{\infty}-\left\|\sigma_{1}^{\varepsilon}\right\|_{\infty}+2 \varepsilon\right\} \\
& \leq \max \left\{\left\|\sigma_{1}^{\varepsilon}\right\|_{\infty}+\left\|\sigma_{2}^{\varepsilon}\right\|_{\infty}-2 \underline{\sigma},\left\|\sigma_{2}^{\varepsilon}\right\|_{\infty}-\underline{\sigma}+2 \varepsilon\right\} .
\end{aligned}
$$

Hence, $\forall \varepsilon \in\left[0, \varepsilon_{0}\right)$ with $\varepsilon_{0} \leq \underline{\sigma} / 4$ :

$$
\left\|\left(\left\|\sigma_{1}^{\varepsilon}\right\|_{\infty}+\left\|\sigma_{2}^{\varepsilon}\right\|_{\infty}-2 \sigma_{i}\right)\right\|_{\infty} \leq \max \left\{\left\|\sigma_{1}^{\varepsilon}\right\|_{\infty}+\left\|\sigma_{2}^{\varepsilon}\right\|_{\infty}-2 \underline{\sigma},\left\|\sigma_{2}^{\varepsilon}\right\|_{\infty}-\underline{\sigma} / 2\right\},
$$

which implies that $\forall \mathbf{x} \in \Omega$ :

$$
\begin{aligned}
& \sqrt{\lambda(\tilde{d}, \sigma)}=\left\|\frac{\frac{\left\|\sigma_{1}^{\varepsilon}\right\|_{\infty}+\left\|\sigma_{2}^{\varepsilon}\right\|_{\infty}}{2}-\sigma}{\frac{\left\|\sigma_{1}^{\varepsilon}\right\|_{\infty}+\left\|\sigma_{2}^{\varepsilon}\right\|_{\infty}}{2}}\right\|_{\infty}=\frac{\left\|\left(\left\|\sigma_{1}^{\varepsilon}\right\|_{\infty}+\left\|\sigma_{2}^{\varepsilon}\right\|_{\infty}-2 \sigma\right)\right\|_{\infty}}{\left\|\sigma_{1}^{\varepsilon}\right\|_{\infty}+\left\|\sigma_{2}^{\varepsilon}\right\|_{\infty}} \\
& \leq \max _{i=1,2} \frac{\left\|\left(\left\|\sigma_{1}^{\varepsilon}\right\|_{\infty}+\left\|\sigma_{2}^{\varepsilon}\right\|_{\infty}-2 \sigma_{i}\right)\right\|_{\infty}}{\left\|\sigma_{1}^{\varepsilon}\right\|_{\infty}+\left\|\sigma_{2}^{\varepsilon}\right\|_{\infty}} \\
& \leq \frac{\max \left\{\left\|\sigma_{1}^{\varepsilon}\right\|_{\infty}+\left\|\sigma_{2}^{\varepsilon}\right\|_{\infty}-2 \underline{\sigma},\left\|\sigma_{2}^{\varepsilon}\right\|_{\infty}-\underline{\sigma} / 2\right\}}{\left\|\sigma_{1}^{\varepsilon}\right\|_{\infty}+\left\|\sigma_{2}^{\varepsilon}\right\|_{\infty}}<1,
\end{aligned}
$$

which yields the required result.

\section{A parametric level set approach}

Since a part information of $\sigma(\mathbf{x})$ is given by (2), it is sufficiently to find $\partial D$ for recovering $\sigma(\mathbf{x})$. Consider $\partial D$ as a zero level set of a Lipschiz continuous function $\phi(\mathbf{x})$. Namely,

$$
\left\{\begin{array}{l}
\phi(\mathbf{x})>0, \quad \mathbf{x} \in D \\
\phi(\mathbf{x})=0, \quad \mathbf{x} \in \partial D \\
\phi(\mathbf{x})<0, \quad \mathbf{x} \in \Omega \backslash D .
\end{array}\right.
$$

Using the Heaviside projector

$$
H(\phi)(\mathbf{x})= \begin{cases}1, & \phi(\mathbf{x})>0 \\ 0, & \phi(\mathbf{x})<0\end{cases}
$$

$\sigma(\mathbf{x})$ can be represented as $\sigma(\mathbf{x})=\sigma_{1}(\mathbf{x}) H(\phi(\mathbf{x}))+\sigma_{2}(\mathbf{x})(1-H(\phi(\mathbf{x})))$. An approximation of $\sigma(\mathbf{x})$ by a polluted data $\left\{\sigma_{1}^{\varepsilon}, \sigma_{1}^{\varepsilon}\right\}$ can be calculated by

$$
\sigma^{\varepsilon}(\mathbf{x})=\sigma_{1}^{\varepsilon}(\mathbf{x}) H(\phi(\mathbf{x}))+\sigma_{2}^{\varepsilon}(\mathbf{x})(1-H(\phi(\mathbf{x}))) .
$$

Hence, using the level set strategy, the optimization problem (10) is substituted by

$$
\min _{\phi} \frac{1}{2}\left\|T^{\sigma^{\varepsilon}(\phi) \epsilon} \nabla u-{ }^{\epsilon} \nabla u\right\|_{L^{2}(\Omega)^{N}}^{2}+\alpha \Psi\left(\sigma^{\varepsilon}(\phi)\right)=: \min _{\phi} \mathcal{J}(\phi),
$$

where function $\sigma^{\varepsilon}(\phi)$ is defined in (17).

Now, let us discuss the method of solving the optimization problem (18). 


\subsection{Computing the gradient of $\mathcal{J}(\phi)$}

It is well known that a minimizer of problem (18) satisfies $\partial \mathcal{J}(\phi) / \partial \phi=0$, which is often solved by the gradient descent method [12]. I.e., we solve the following ordinary differential equation to the steady state

$$
\phi_{t}+\partial \mathcal{J}(\phi) / \partial \phi=0 \text {. }
$$

Remark 1. Note that for the evolution approach (19) it is desired to initialize a minimization process with some level set function $\phi_{0}$ and evolve the function to find a $\phi$ which minimizes the functional $\mathcal{J}$. To take into account the concept of evolution, an artificial time is defined where the level set function at every time frame $t \geq 0$ is rewritten as $\phi(\mathbf{x}, t)$ and the zero level set of $\phi(\mathbf{x}, t)$ is denoted as $\partial D_{t}$.

First, look at the computation of the gradient of $\mathcal{J}(\sigma)$ with respect to $\sigma$ in the direction $\delta \sigma$, i.e.

$$
\begin{gathered}
\frac{\partial \mathcal{J}}{\partial \sigma}[\delta \sigma]=\left\langle\frac{\partial T^{\sigma} \epsilon \nabla u}{\partial \sigma}[\delta \sigma], T^{\sigma \epsilon} \nabla u-{ }^{\epsilon} \nabla u\right\rangle_{L^{2}(\Omega)^{N}}+\alpha \frac{\partial \Psi(\sigma)}{\partial \sigma}[\delta \sigma] \\
=\left\langle M, T^{\sigma}{ }^{\epsilon} \nabla u-{ }^{\epsilon} \nabla u\right\rangle_{L^{2}(\Omega)^{N}}+\alpha \frac{\partial \Psi(\sigma)}{\partial \sigma}[\delta \sigma]
\end{gathered}
$$

where $M \in L^{2}(\Omega)^{N}$ satisfies $\int_{\Omega} \tilde{d} \cdot M \cdot \nabla v d \mathbf{x}=-\int_{\Omega} \delta \sigma^{\epsilon} \nabla u \cdot \nabla v d \mathbf{x}$ for all $v \in H_{0}^{1}(\Omega)$. Therefore,

$$
\begin{aligned}
\frac{\partial \mathcal{J}}{\partial \sigma}[\delta \sigma] & =\tilde{d}^{-1} \cdot\left\langle\tilde{d} \cdot M, T^{\sigma \epsilon} \nabla u-{ }^{\epsilon} \nabla u\right\rangle_{L^{2}(\Omega)^{N}}+\alpha \frac{\partial \Psi(\sigma)}{\partial \sigma}[\delta \sigma] \\
& =\tilde{d}^{-1} \cdot\left\langle-\delta \sigma{ }^{\epsilon} \nabla u,\left(T^{\sigma \epsilon} \nabla u-{ }^{\epsilon} \nabla u\right)\right\rangle_{L^{2}(\Omega)^{N}}+\alpha \frac{\partial \Psi(\sigma)}{\partial \sigma}[\delta \sigma] . \\
& =-\tilde{d}^{-1} \cdot\left\langle\delta \sigma,{ }^{\epsilon} \nabla u \cdot\left(T^{\sigma}{ }^{\epsilon} \nabla u-{ }^{\epsilon} \nabla u\right)\right\rangle_{L^{2}(\Omega)}+\alpha \frac{\partial \Psi(\sigma)}{\partial \sigma}[\delta \sigma],
\end{aligned}
$$

which implies

$$
\frac{\partial \mathcal{J}}{\partial \sigma}=-\tilde{d}^{-1} \cdot{ }^{\epsilon} \nabla u \cdot\left(T^{\sigma}{ }^{\epsilon} \nabla u-{ }^{\epsilon} \nabla u\right)+\alpha \frac{\partial \Psi(\sigma)}{\partial \sigma}
$$

Now, let us discuss the regularization term in (5). From now on, assume that $\sigma \in B V(\Omega)$, where $B V(\Omega) \subset L^{\infty}(\Omega)$ denotes the spaces of functions of bounded variation $[25]$. Define

$$
\Psi(\sigma)=\int_{\Omega}|\nabla \sigma| d \mathbf{x}=\int_{\Omega}\left|\sigma_{1}-\sigma_{2}\right| \cdot \mathfrak{D}(\phi) \cdot|\nabla \phi| d \mathbf{x}
$$

which penalizes the product of the jump between different regions and the arc length of their interfaces. Here, $\mathfrak{D}(\cdot)$ denotes the Dirac function and $|\nabla \phi|=\left(\sum_{|\nu|=1}\left(\partial^{\nu} \phi\right)^{2}\right)^{\frac{1}{2}}$, where $\nu=\left(\nu_{1}, \ldots, \nu_{N}\right)$ is a multi-index of order $|\nu|=\nu_{1}+\ldots+\nu_{N}$, and $\partial^{\nu} \equiv \frac{\partial^{\nu_{1}}}{\partial x_{1}^{\nu_{1}}} \cdots \frac{\partial^{\nu_{N}}}{\partial x_{N}^{\nu_{N}}}$.

Define an approximate regularization term as

$$
\Psi_{\varepsilon}(\sigma)=\int_{\Omega}\left|\sigma_{1}^{\varepsilon}-\sigma_{2}^{\varepsilon}\right| \cdot \mathfrak{D}(\phi) \cdot|\nabla \phi| d \mathbf{x},
$$


where the approximate data $\left\{\sigma_{1}^{\varepsilon}, \sigma_{2}^{\varepsilon}\right\}$ is defined by (3).

Denote a set of a priori information of solution as:

$$
\Theta=\left\{\sigma \in B V(\Omega): \sigma(\mathbf{x})=\left\{\begin{array}{ll}
\sigma_{1}(\mathbf{x}) & \text { when } \mathbf{x} \in D \subset \Omega, \\
\sigma_{2}(\mathbf{x}) & \text { when } \mathbf{x} \in \Omega \backslash D
\end{array}\right\} .\right.
$$

Lemma 4. For any $\sigma \in \Theta,\left|\Psi_{\varepsilon}(\sigma)-\Psi(\sigma)\right| \leq C_{\phi} \cdot \varepsilon$, where constant $C_{\phi}$ only depends on the test function $\phi$ and domain $\Omega$.

Proof. Fix $\sigma \in \Theta$, by the following sequence of inequalities

$$
\begin{aligned}
& \left|\Psi_{\varepsilon}(\sigma)-\Psi(\sigma)\right| \\
& =\left|\int_{\Omega}\right| \sigma_{1}^{\varepsilon}-\sigma_{2}^{\varepsilon}|\cdot \mathfrak{D}(\phi) \cdot| \nabla \phi\left|d \mathbf{x}-\int_{\Omega}\right| \sigma_{1}-\sigma_{2}|\cdot \mathfrak{D}(\phi) \cdot| \nabla \phi|d \mathbf{x}| \\
& \leq \int_{\Omega}|| \sigma_{1}^{\varepsilon}-\sigma_{2}^{\varepsilon}|-| \sigma_{1}-\sigma_{2}|| \cdot \mathfrak{D}(\phi) \cdot|\nabla \phi| d \mathbf{x} \\
& \leq \int_{\Omega}\left|\sigma_{1}^{\varepsilon}-\sigma_{2}^{\varepsilon}-\sigma_{1}+\sigma_{2}\right| \cdot \mathfrak{D}(\phi) \cdot|\nabla \phi| d \mathbf{x} \\
& \leq \int_{\Omega}\left\{\left|\sigma_{1}^{\varepsilon}-\sigma_{1}\right|+\left|\sigma_{2}^{\varepsilon}-\sigma_{2}\right|\right\} \cdot \mathfrak{D}(\phi) \cdot|\nabla \phi| d \mathbf{x} \\
& \leq\left\{\left\|\sigma_{1}^{\varepsilon}-\sigma_{1}\right\|_{\infty}+\left\|\sigma_{2}^{\varepsilon}-\sigma_{2}\right\|_{\infty}\right\} \cdot \int_{\Omega} \mathfrak{D}(\phi) \cdot|\nabla \phi| d \mathbf{x} \leq 2 \varepsilon \int_{\Omega} \mathfrak{D}(\phi) \cdot|\nabla \phi| d \mathbf{x}
\end{aligned}
$$

we obtain the required result with the constant $C_{\phi}=2 \int_{\Omega} \mathfrak{D}(\phi) \cdot|\nabla \phi| d \mathbf{x}$.

Now, for a given $\phi$, using the chain rule and the equation (20), one can obtain the derivative of $\mathcal{J}(\phi)$ with respect to $\phi$ :

$$
\frac{\partial \mathcal{J}}{\partial \phi}=-\tilde{d}^{-1} \cdot{ }^{\epsilon} \nabla u \cdot\left(T^{\sigma}{ }^{\epsilon} \nabla u-{ }^{\epsilon} \nabla u\right)\left(\sigma_{1}^{\epsilon}-\sigma_{2}^{\epsilon}\right) \mathfrak{D}(\phi)-\alpha\left|\sigma_{1}^{\epsilon}-\sigma_{2}^{\epsilon}\right| \nabla \cdot\left(\frac{\nabla \phi}{|\nabla \phi|}\right) \mathfrak{D}(\phi) .
$$

Finally, we rewrite evolution approach (19) as

$$
\frac{\partial \phi(\mathbf{x}, t)}{\partial t}=v(\mathbf{x}, t)
$$

where, $v=\tilde{d}^{-1} \cdot{ }^{\epsilon} \nabla u \cdot\left(T^{\sigma \epsilon} \nabla u-{ }^{\epsilon} \nabla u\right)\left(\sigma_{1}^{\epsilon}-\sigma_{2}^{\epsilon}\right)+\alpha\left|\sigma_{1}^{\epsilon}-\sigma_{2}^{\epsilon}\right| \nabla \cdot\left(\frac{\nabla \phi}{|\nabla \phi|}\right)$.

Remark 2. Here, we get $v$ by omitting Dirac function $\mathfrak{D}$ from $-\partial \mathcal{J} / \partial \phi$ since $\mathfrak{D}(\phi)$ is always positive, which implies that $v$ is still a descent direction (various numerical experiments indicate that such reduction works even better).

\subsection{Calculating the level set function $\phi(\mathbf{x}, t)$}

Using the compact support radial basis functions (CSRBFs), which bypass many difficulties with traditional level set methods such as reinitialization and use of signal distance function [19], we expand $\phi(\mathbf{x}, t)$ as (here, we separate space and time variable of the level set function)

$$
\phi(\mathbf{x}, t)=\sum_{i=1}^{n} \beta_{i}(t) \cdot \psi\left(\left\|\mathbf{x}-\mathbf{x}_{i}\right\|\right)=: \Phi(\mathbf{x}) \cdot \beta(t),
$$


where $\psi$ is a CSRBF and $\left\{\mathbf{x}_{i}\right\}_{i=1, \ldots, n}$ are centers (both of them are given in practice).

Substituting (25) into (24) yields the following ordinary differential equation

$$
\Phi(\mathbf{x}) \frac{d \beta(t)}{d t}=v(\mathbf{x}, t)
$$

Setting RBFs centers as collocation points, we discretize (26) as

$$
A \frac{d \beta(t)}{d t}=V
$$

where $A$ is a strictly positive definite matrix of size $n \times n$ with $A_{i j}=\psi\left(\left\|\mathbf{x}_{i}-\mathbf{x}_{j}\right\|\right)$, $i, j=\overline{1, n}$, and $V$ is a vector of size $N \times 1$ with $V_{i}=v\left(\mathbf{x}_{i}, t\right)$.

Applying the forward Euler's method to (27) yields

$$
A \frac{\beta^{k+1}-\beta^{k}}{\tau^{k}}=V^{k}
$$

where $\tau^{k}$ is the step size and $\beta^{k}$ denotes the solution vector at the moment $t_{k}$. Here, $V_{i}^{k}=v\left(\mathbf{x}_{i}, t^{k}\right)$. For the sake of stability of the evolution, the time step $\tau^{k}$ is computed as

$$
\tau^{k}=\varsigma \frac{h}{\max _{i}\left|V_{i}^{k}\right|},
$$

where $0<\varsigma<1$ and $h$ is uniform mesh step.

Remark 3. (a) Without considering the time cost one can use the line search for choosing an optimal parameter $\varsigma$. (b) Since $A$ is a positive definite symmetric matric, one can use a LDLT or a Cholesky decomposition to solve the equation.

For realization, in this work, we apply the Wendlan's CSRBFs with $C^{2}$ smoothness [26], namely,

$$
\psi\left(\mu\left\|\mathbf{x}-\mathbf{x}_{i}\right\|\right)=\psi(r)= \begin{cases}(1-r)^{3}(3 r+1), & 0 \leq r \leq 1 \\ 0, & r>1\end{cases}
$$

We refer to $s=1 / \mu$ the support size of $\psi$. Aiming to get the sparse interpolation matrix $A, s$ must be proper chosen [27]. Indeed, the value of $s$ plays the role of regularization parameter in the problem. If $s$ is chosen too small, the level set function $\phi$ will not be continuous. Whereas if $s$ is too large, $A$ will become dense and we loose the interest of using CSRBFs due to the high computational cost. In this work, we choose $s=0.1$.

In the end of this section, we indicate that one can exactly and efficiently calculate $\nabla \cdot\left(\frac{\nabla \phi}{|\nabla \phi|}\right)$ as a result of parametrization of the level set function. Denote $\mathbf{x}=(x, y)$. By a simple deduction, we acquire

$$
\nabla \cdot(\nabla \phi /|\nabla \phi|)=\left(\phi_{x x} \phi_{y}^{2}+\phi_{y y} \phi_{x}^{2}-2 \phi_{x} \phi_{y} \phi_{x y}\right) /|\nabla \phi|^{3} .
$$

Note that for the chosen CSRBFs in (30), we have $\psi_{x}=-12(1-r)^{2}\left(x-x_{0}\right), \psi_{y}=$ $-12(1-r)^{2}\left(y-y_{0}\right), \psi_{x y}=24 \frac{(1-r)}{r}\left(x-x_{0}\right)\left(y-y_{0}\right), \quad \psi_{x x}=24 \frac{(1-r)}{r}\left(x-x_{0}\right)^{2}-12(1-r)^{2}$ 
and $\psi_{y y}=24 \frac{(1-r)}{r}\left(y-y_{0}\right)^{2}-12(1-r)^{2}$, where $\left(x_{0}, y_{0}\right)$ is center of CSRBF $\psi$. According to $\phi=\sum_{i=1}^{n} \beta_{i} \psi_{i}$, we obtain

$$
\nabla \cdot\left(\frac{\nabla \phi}{|\nabla \phi|}\right)(\mathbf{x})=\frac{A_{4} \beta \cdot\left(A_{2} \beta\right)^{2}+A_{5} \beta \cdot\left(A_{1} \beta\right)^{2}-2 A_{1} \beta \cdot A_{2} \beta \cdot A_{3} \beta}{\left(\sqrt{A_{1} \beta+A_{2} \beta}\right)^{3}},
$$

where $\mathbf{x}=\left\{\mathbf{x}_{i}\right\}_{i=1}^{n}$ are centers of these CSRBFs and $A_{1}, A_{2}, A_{3}, A_{4}, A_{5}$ are matrixes of size $n \times n$, satisfying $A_{1, i j}=\psi_{i, x}\left(\mathbf{x}_{j}\right), A_{2, i j}=\psi_{i, y}\left(\mathbf{x}_{j}\right), A_{3, i j}=\psi_{i, x y}\left(\mathbf{x}_{j}\right), A_{4, i j}=$ $\psi_{i, x x}\left(\mathbf{x}_{j}\right)$ and $A_{5, i j}=\psi_{i, y y}\left(\mathbf{x}_{j}\right), i, j=\overline{1, n}$.

\section{Two approaches to estimate $\nabla u$ by the error data $u^{\eta}$}

In practice, sometimes, it is easy to measure the data $u^{\eta}$ instead of ${ }^{\epsilon} \nabla u$. Obviously, $\nabla u^{\eta}$ in itself usually share very little similarity with $\nabla u$ especially for multiscale/homogenization problems. Hence, the purpose in this section is to propose two effective approximations of $\nabla u$ by the noisy data $u^{\eta}$. Denote $M\left(u^{\eta}\right)$ as the stabilized solution data of $u^{\eta}$ such that $T V\left(M\left(u^{\eta}\right)-u\right) \leq T V\left(u^{\eta}-u\right)$. Then, obviously, $M\left(u^{\eta}\right)$ is a better approximation than $u^{\eta}$ for estimating $\nabla u$.

\subsection{Approach I}

For simplicity, we fix the domain $\Omega=(0,1)^{2}$, and assume that the grid is uniform. Suppose that $u=u(x, y)$ is a smooth function on $\Omega$ and noisy samples $u_{i, j}^{\eta}$ of the values $u\left(x_{i}, y_{j}\right)$ are known at the points of a uniform grid $\left\{x_{i}, y_{j}\right\}_{i, j=1}^{n}$. Let $h=x_{i+1}-x_{i}=$ $y_{i+1}-y_{i}=1 / n$ be the mesh size of the grid and suppose that $\left|u_{i, j}^{\eta}-u\left(x_{i}, y_{j}\right)\right| \leq \eta$, where $\eta$ is a known level of noise in data. We are interested in finding a smooth approximation $M\left(\nabla u^{\eta}\right)$ of $\nabla u$ from the given noisy data $\left\{u_{i}^{\eta}\right\}$, with some guaranteed (best possible) accuracy.

Let $M\left(\nabla u^{\eta}\right)=\nabla u_{s}$, where $u_{s}$ is the minimizer of the following optimization problem

$$
\min _{u_{s} \in S P(\Omega)} \frac{1}{n^{2}} \sum_{i, j}^{n}\left(u_{i, j}^{\eta}-u_{s}\left(x_{i}, y_{j}\right)\right)^{2}+\alpha_{s}\left\|\triangle u_{s}\right\|_{L^{2}(\Omega)}^{2},
$$

where $S P(\Omega)$ represents the set of all cubic spline over $\Omega$, and regularization parameter $\alpha_{s}$ is chosen by the discrepancy principle, i.e., $\alpha_{s}$ is selected such that the minimizing element $u_{s}^{\alpha_{s}}$ of (32) satisfies

$$
\frac{1}{n^{2}} \sum_{i, j}^{n}\left(u_{i, j}^{\eta}-u_{s}^{\alpha_{s}}\left(x_{i}, y_{j}\right)\right)^{2}=\eta^{2}
$$

Using the 1D result in [28], it is not difficult to prove the following theorem.

Theorem 5. Let $u \in H^{2}(\Omega)$ and let $u_{s}^{*}$ be the minimizer of problem (32). Then

$$
\left\|\nabla u_{s}^{*}-\nabla u\right\|_{L^{2}(\Omega)^{2}} \leq c\left(h\|\Delta u\|_{L^{2}(\Omega)}+\sqrt{\eta}\|\Delta u\|_{L^{2}(\Omega)}^{1 / 2}\right)
$$

where $c$ is a constant. 
The above theorem says that, as long as $h>\sqrt{\eta}\|\Delta u\|_{L^{2}(\Omega)}^{1 / 2}$, the error bound is of the same order as that for finite differences (combining consistency error and propagation error for one-sided finite differences, one arrives at the bound: $\left|\frac{u_{i+1, j}^{\eta}-u_{i, j}^{\eta}}{h}-\frac{\partial u(x, y)}{\partial x}\right| \leq$ $c(h+\eta / h))$. However, the bound in Theorem 5 remains of order $\sqrt{\eta}$ when $h \rightarrow 0$. Moreover, the error estimate in Theorem $\mathbf{5}$ is sharp in the sense that for $\eta=0$, i.e., noise-free data, the right-hand side coincides up to a multiplicative constant with the best-possible worst case bound for the interpolating spline, see [28]. Therefore, the gradient of the minimizer of $(32)$, say $\nabla u_{s}$, can be used as a smooth approximation of $\nabla u$.

It should be noted that the assumption $u \in H^{2}$ in Theorem 5 holds in many cases for PDEs with the discontinuous coefficient. For example, as shown in the simulation section later, for model problems, in which the source term $f$ is obtained by the given discontinuous coefficient $\sigma$ and smooth $u$ or $\nabla u$, condition $u \in H^{2}$ holds obviously. Furthermore, if we consider the finite element approximate solution $u_{e}=\Sigma c_{i} \phi_{i}$, where $\left\{\phi_{i}\right\}$ is the basis in the finite element space (of piecewise linear elements in our simulations) on the triangulation, then $u_{e} \in H^{2}$ obviously. This means that if we consider the weak solution (or variational formulation) in finite element space, it always holds $u \in H^{2}$.

\subsection{Approach II}

Now, consider another method of estimating $\nabla u$. Obviously, for periodical problems, $M\left(u^{\eta}\right)$ can be computed as an average of $u^{\eta}$ over a neighborhood of $u^{\eta}$ at a fixed point; i.e., $\forall x_{0} \in \Omega, M\left(u^{\eta}\right)\left(x_{0}\right):=\frac{1}{\mu\left(\mathcal{O}_{x_{0}}\right)} \int_{\mathcal{O}_{x_{0}}} u^{\eta}(x) d x$, where $\mathcal{O}_{x_{0}}$ is a neighborhood of $x_{0}$ and $\mu(\cdot)$ denotes the Lebesgue measure. For instance, for a two-dimensional $2 \delta$-periodic case, $M\left(u^{\eta}\right)$ can be defined by

$$
M\left(u^{\eta}\right)\left(x_{0}\right):=4 \delta^{2} \int_{x_{1,0}-\delta}^{x_{1,0}+\delta} \int_{x_{2,0}-\delta}^{x_{2,0}+\delta} u^{\eta}\left(x_{1}, x_{2}\right) d x_{1} d x_{2},
$$

$\forall x_{0} \equiv\left(x_{1,0}, x_{2,0}\right) \in \Omega$. Then, $\nabla M\left(u^{\eta}\right)$ can be used as an approximation of $\nabla u$. In this work, to deal with non-periodic cases we apply analytic averaging techniques in $[29,30]$ : By defining an effective approximation of $u$ as

$$
\hat{u}\left(x_{0}\right):=\lim _{\delta \rightarrow 0}\left(\lim _{\eta \rightarrow 0} \frac{1}{\mu\left(\mathcal{O}_{\delta}\right)} \int_{\mathcal{O}_{\delta}\left(x_{0}\right)} u^{\eta}(x) d x\right),
$$

where $\mathcal{O}_{\delta}\left(x_{0}\right)$ is a neighborhood of $x_{0}$ with diameter $\delta$. Using the $1 \mathrm{D}$ result in [29], it is possible to prove the following theorem.

Theorem 6. Assume that the eigenvalues $\lambda_{\eta}^{n}(x)$ of the Jacobian of $\nabla u^{\eta}$ satisfy the following conditions for any $x \in \mathbb{R}_{+}^{N}$ : (1) $R e \lambda_{\eta}^{n} \leq \hat{C}_{1}, 1 \leq n \leq N$, (2) there is $n_{0} \geq 1$ such that $\left|\lambda_{\eta}^{n}\right| \leq \hat{C}_{2}$, for $1 \leq n \leq n_{0} \leq N$ and $\hat{C}_{3} \leq \eta\left|\lambda_{\eta}^{n}\right| \leq \hat{C}_{4}$, for $n_{0}<n \leq N$; here $\hat{C}_{1,2,3,4}$ are constants; (2) $\min _{n_{1}, n_{2}}\left|\lambda_{\eta}^{n_{1}}-\lambda_{\eta}^{n_{2}}\right|>\rho>0, n_{1}<n_{0}$ and $n_{2}>n_{0}$. Let $s$ be the largest integer such that $\left|\partial^{\nu} \hat{u}(x)\right| \leq \tilde{C}$ for $0 \leq|\nu| \leq s$, where $\tilde{C}$ is a constant independent of $\eta$. Denote $x^{r}=x_{1}^{r_{1}} \cdots x_{N}^{r_{N}}$ and let the kernel $K(x)$ be defined as

$$
\int_{\Omega} K(x) x^{r} d x= \begin{cases}1 & |r|=0 \\ 0 & 1 \leq|r| \leq|\nu|\end{cases}
$$


Assume $\delta \rightarrow 0$ when $\eta \rightarrow 0$. Denote $K_{\delta}(x):=\frac{1}{\delta} K\left(\frac{x}{\delta}\right)$. Then,

1. $K_{\delta} * u^{\eta}$ approximates (33), i.e., $K_{\delta} * u^{\eta} \rightarrow \hat{u}$ strongly in $L^{2}(\Omega)$ as $\eta \rightarrow 0$,

2. $K_{\delta} * \nabla u^{\eta} \rightarrow \nabla \hat{u}$ strongly in $L^{2}(\Omega)^{N}$ as $\eta \rightarrow 0$.

By above theorem, one can estimate an effective approximation of $\nabla u$ by

$$
M\left(\nabla u^{\eta}\right)\left(x_{0}\right)=K_{\delta} * \nabla u^{\eta}=\int_{\mathcal{O}_{\delta}\left(x_{0}\right)} K_{\delta}\left(x-x_{0}\right) \nabla u^{\eta}(x) d x
$$

In our finite element approximation, $\mathcal{O}_{\delta}\left(x_{0}\right)$ denotes the triangles, distant between whose vertexes or centroids and point $x_{0}$ is no more than $\delta$. Then, the discretization of (34) in our finite element approximation is

$$
M(\nabla u)\left(x_{0}\right)=K_{\delta} * \nabla u^{\eta} \approx \frac{\pi \delta^{2}}{4|\Lambda|} \sum_{\mu_{i} \in \Lambda} K_{\delta}\left(\mu_{i}-x_{0}\right) \nabla u^{\eta}\left(\mu_{i}\right),
$$

where $\Lambda=\left\{\mu_{i} \in \mathcal{G}:\left\|\mu_{i}-x_{0}\right\|_{2} \leq \delta / 2\right\}(|\Lambda|$ denotes as its cardinality) and $\mathcal{G}=\mathcal{G}(\mathcal{T})$ is the set of all centroids of triangles of a triangulation $\mathcal{T}$ of domain $\Omega$. For problems with a symmetric/asymmetric structure, it is reasonable to use a symmetric/asymmetric kernel. A typical choice for symmetric kernels with support on the unit ball is the exponential kernel $K_{1}=C_{a} \exp \left(c_{a} /\left(|x|^{2}-1\right)\right)$, where $|x|=\sum_{i}^{N} x_{i}^{2}, c_{a}$ is a positive constant, and $C_{a}$ is the normalization constants.

Remark 4. By above two approaches, a constant $C$ exists such that $\| M\left(\nabla u^{\eta}\right)-$ $\nabla \bar{u} \|_{L^{2}(\Omega)^{N}} \leq C \eta$. In practice, we approximate the whole value of $C \eta$ as the required error level $\epsilon$ in the estimate (4).

\section{Regularization property of the method}

In order to systematically analyze the stability property of the developed method, we reformulate the initial problem (1) as an operator equation. Define an operator $A: L^{\infty}(\Omega) \rightarrow L^{2}(\Omega)^{N}$ such that $\forall \sigma \in L^{\infty}(\Omega)$ :

$$
A \sigma:=T^{\sigma} \nabla u
$$

where operator $T^{\sigma}$ is defined in (9). Then, the inverse problem for (1) becomes to solve an operator equation

$$
A \sigma=\nabla u, \quad \sigma \in \Theta,
$$

where $\Theta$ defined in (23).

Assume that the solution set $\Sigma^{*}$ of $(37)$ is nonempty. For many situations $\Sigma^{*}$ may contain more than one element. In this case, using the auxiliary functional $\Psi(\cdot)$, we select the so-called $\Psi$-optimal solutions to (37), i.e., functions $\bar{\sigma}(\mathbf{x}) \in \Sigma^{*}$ such that

$$
\Psi(\bar{\sigma})=\inf _{\sigma \in \Sigma^{*}} \Psi(\sigma)=: \bar{\Psi} .
$$

Denote the set of these solutions by $\bar{\Sigma}$. Then $\bar{\Psi}=\Psi(\bar{\Sigma})$.

Suppose that instead of the exact data $\nabla u$ we only know a polluted one ${ }^{\epsilon} \nabla u$ such that the inequality (4) holds (if we are only given the approximate data $u^{\eta}$, 
then, calculate ${ }^{\epsilon} \nabla u=M\left(\nabla u^{\eta}\right)$, where $M\left(\nabla u^{\eta}\right)$ is the smooth approximation of $\nabla u$, introduced in Section 4). Define approximate operator $A_{\epsilon}$ of $A$ such that

$$
A_{\epsilon} \sigma:=T^{\sigma \epsilon} \nabla u \text {. }
$$

Furthermore, instead of exact information set $\Theta$ we are given only polluted one, whose finite element reduction is:

$$
\begin{aligned}
& \widehat{\Theta}_{\varepsilon}=\{\sigma \in B V(\Omega): \\
& \left.\quad \sigma(\mathbf{x})=\sigma_{1}^{\varepsilon}(\mathbf{x}) H_{L}(\phi(\mathbf{x}))+\sigma_{2}^{\varepsilon}(\mathbf{x})\left(1-H_{L}(\phi(\mathbf{x}))\right), \phi=\sum_{i=1}^{n} \beta_{i} \psi_{i} .\right\},
\end{aligned}
$$

where $H_{L}(\phi)=\pi^{-1} \tan ^{-1}(L \cdot \phi)+0.5$ is an approximation of Heaviside projection. Here $\mathrm{L}$ is a fixed sufficiently large number. Then, the optimization problem (10) becomes

$$
\min _{\sigma \in \widehat{\Theta}_{\varepsilon}} \frac{1}{2}\left\|A_{\epsilon} \sigma-{ }^{\epsilon} \nabla u\right\|_{L^{2}(\Omega)^{N}}^{2}+\alpha \Psi_{\varepsilon}(\sigma) .
$$

Denote $\sigma_{\varepsilon, \epsilon}^{\alpha(\varepsilon, \epsilon)}$ as a minimizer of (40). The purpose in the section is to prove the convergence property of the obtained solution $\sigma_{\varepsilon, \epsilon}^{\alpha(\varepsilon, \epsilon)}$, i.e., $\sigma_{\varepsilon, \epsilon}^{\alpha(\varepsilon, \epsilon)} \rightarrow \bar{\Sigma}$ in $L^{\infty}(\Omega)$ as $(\varepsilon, \epsilon) \rightarrow 0$.

As demonstrated in [31, 32], the following theorem holds

Theorem 7. Assume the following conditions hold

1) $A$, defined in (36), and $A_{\epsilon}$, defined in (38), are continue operators, acting from $L^{\infty}(\Omega)$ into $L^{2}(\Omega)^{N}$.

2) $\forall \sigma \in \Theta$ or $\widehat{\Theta}_{\varepsilon}:\left\|A_{\epsilon} \sigma-A \sigma\right\| \leq \Upsilon(\epsilon, \Psi(\sigma))$, where function $\Upsilon(s, t)$ satisfies the following conditions 2.1) it is a continuous function for $s, t \geq 0$; 2.2) for any fixed $s>0$ it is a monotonically nondecreasing function with respect to $t$; 2.3) $\forall s, t>0: \Upsilon(s, t)>0$; 2.4) $\forall t>0: \Upsilon(0, t)=0$ and 2.5) for any fixed $s>0$ : $\Upsilon(s, t) \rightarrow+\infty$ as $t \rightarrow+\infty$;

3) $\Psi$, defined in (21), satisfies the following two conditions:

3.1) Functional $\Psi(\cdot)$ is lower semicontinuous on $\widehat{\Theta}_{\varepsilon}$ (defined in (39)) with respect to the $L^{\infty}(\Omega)$ norm topology.

3.2) The nonempty sets $\Psi_{C}:=\left\{\sigma \in \widehat{\Theta}_{\varepsilon}: \Psi(\sigma) \leq C\right\}$ are compact sets in $L^{\infty}(\Omega)$.

3.3) $\forall \sigma \in \Theta$ or $\widehat{\Theta}_{\varepsilon}$, there exists a positive number $\varepsilon_{0}$ such that $\forall \varepsilon \in\left(0, \varepsilon_{0}\right]$ : $\left|\Psi_{\varepsilon}(\sigma)-\Psi(\sigma)\right| \leq \varepsilon$, where $\Psi_{\varepsilon}$ is defined in (22).

4) The choice of regularization parameter $\alpha$ is done so as to guarantee the so-called regularity conditions

$$
\limsup _{(\varepsilon, \epsilon) \rightarrow 0} \Psi_{\varepsilon}\left(\sigma_{\varepsilon, \epsilon}^{\alpha(\varepsilon, \epsilon)}\right) \leq \Psi(\bar{\Sigma})
$$

and

$$
\lim _{(\varepsilon, \epsilon) \rightarrow 0}\left\|A_{\epsilon} \sigma_{\varepsilon, \epsilon}^{\alpha(\varepsilon, \epsilon)}-{ }^{\epsilon} \nabla u\right\|_{L^{2}(\Omega)^{N}}^{2}=\|A \bar{\sigma}-\nabla u\|_{L^{2}(\Omega)^{N}}^{2} .
$$


Then $\sigma_{\varepsilon, \epsilon}^{\alpha(\varepsilon, \epsilon)} \rightarrow \bar{\Sigma}$ in $L^{\infty}(\Omega)$ as $(\varepsilon, \epsilon) \rightarrow 0$.

By the above theorem, it is clear that in order to obtain the convergence property of obtained approximate solution $\sigma_{\varepsilon, \epsilon}^{\alpha(\varepsilon, \epsilon)}$ it is sufficient to check all conditions in Theorem 7.

Denote an admissible error level as

$$
\omega(\varepsilon, \epsilon)=\tilde{d}^{-1} \cdot\left(\varepsilon+\max \left\{\left\|\sigma_{1}^{\varepsilon}\right\|_{\infty},\left\|\sigma_{2}^{\varepsilon}\right\|_{\infty}\right\}\right) \cdot \epsilon
$$

Lemma 8. There exists a number $\varepsilon_{0}$ such that $\forall \varepsilon \in\left(0, \varepsilon_{0}\right]: \omega(\varepsilon, \epsilon) \leq 4 \epsilon$.

Proof. Define $\varepsilon_{0}=\left\|\sigma_{1}^{\varepsilon}\right\|_{\infty}+\left\|\sigma_{2}^{\varepsilon}\right\|_{\infty}$. Then, by definition of $\tilde{d}, \forall \varepsilon \in\left(0, \varepsilon_{0}\right]$

$$
\begin{aligned}
& \tilde{d}^{-1} \cdot\left(\varepsilon+\max \left\{\left\|\sigma_{1}^{\varepsilon}\right\|_{\infty},\left\|\sigma_{2}^{\varepsilon}\right\|_{\infty}\right\}\right) \cdot \epsilon \\
& \leq \frac{2}{\left\|\sigma_{1}^{\varepsilon}\right\|_{\infty}+\left\|\sigma_{2}^{\varepsilon}\right\|_{\infty}}\left(\varepsilon+\left\|\sigma_{1}^{\varepsilon}\right\|_{\infty}+\left\|\sigma_{2}^{\varepsilon}\right\|_{\infty}\right) \cdot \epsilon \\
& \leq \frac{2}{\left\|\sigma_{1}^{\varepsilon}\right\|_{\infty}+\left\|\sigma_{2}^{\varepsilon}\right\|_{\infty}} \cdot 2 \cdot\left(\left\|\sigma_{1}^{\varepsilon}\right\|_{\infty}+\left\|\sigma_{2}^{\varepsilon}\right\|_{\infty}\right) \cdot \epsilon=4 \epsilon
\end{aligned}
$$

which yields the required inequality.

Define a data-to-noise number as

$$
\varpi=\min \left\{\left\|A_{\epsilon} \sigma_{1}^{\varepsilon}-{ }^{\epsilon} \nabla u\right\|_{L^{2}(\Omega)^{N}}^{2},\left\|A_{\epsilon} \sigma_{2}^{\varepsilon}-{ }^{\epsilon} \nabla u\right\|_{L^{2}(\Omega)^{N}}^{2}\right\} .
$$

Theorem 9. Conditions 1)- 3) in Theorem 7 hold for our case. In addition, if the data-to-noise number is greater than the admissible error level, i.e., $\varpi>\omega(\varepsilon, \epsilon)$ and the regularization parameter $\alpha$ is chosen as a positive root of the following generalized discrepancy function

$$
\chi(\alpha)=\left\|A_{\epsilon} \sigma_{\varepsilon, \epsilon}^{\alpha(\varepsilon, \epsilon)}-{ }^{\epsilon} \nabla u\right\|_{L^{2}(\Omega)^{N}}^{2}-\omega(\varepsilon, \epsilon),
$$

then, the regularity conditions (41) and (42) in Theorem 7 are also fulfilled.

The proof of Theorem 9 can be found in the Appendix A. Note that it is not difficult to show that the reconstructed conductivity coefficient $\sigma_{\varepsilon, \epsilon}^{\alpha(\varepsilon, \epsilon)}$, obtained by optimization problem (40) with the generalized discrepancy principle parameter choice rule, has an order optimal property if we a priori know that the exact conductivity coefficient has a property of the representation of sources [31, 33].

\section{A solution algorithm of the parameter identification problem in PDEs}

In this section, let us describe steps of the solution algorithm of the collage method for solving inverse problem (1). This algorithm (Algorithm 1) is based on the theory mentioned above. 


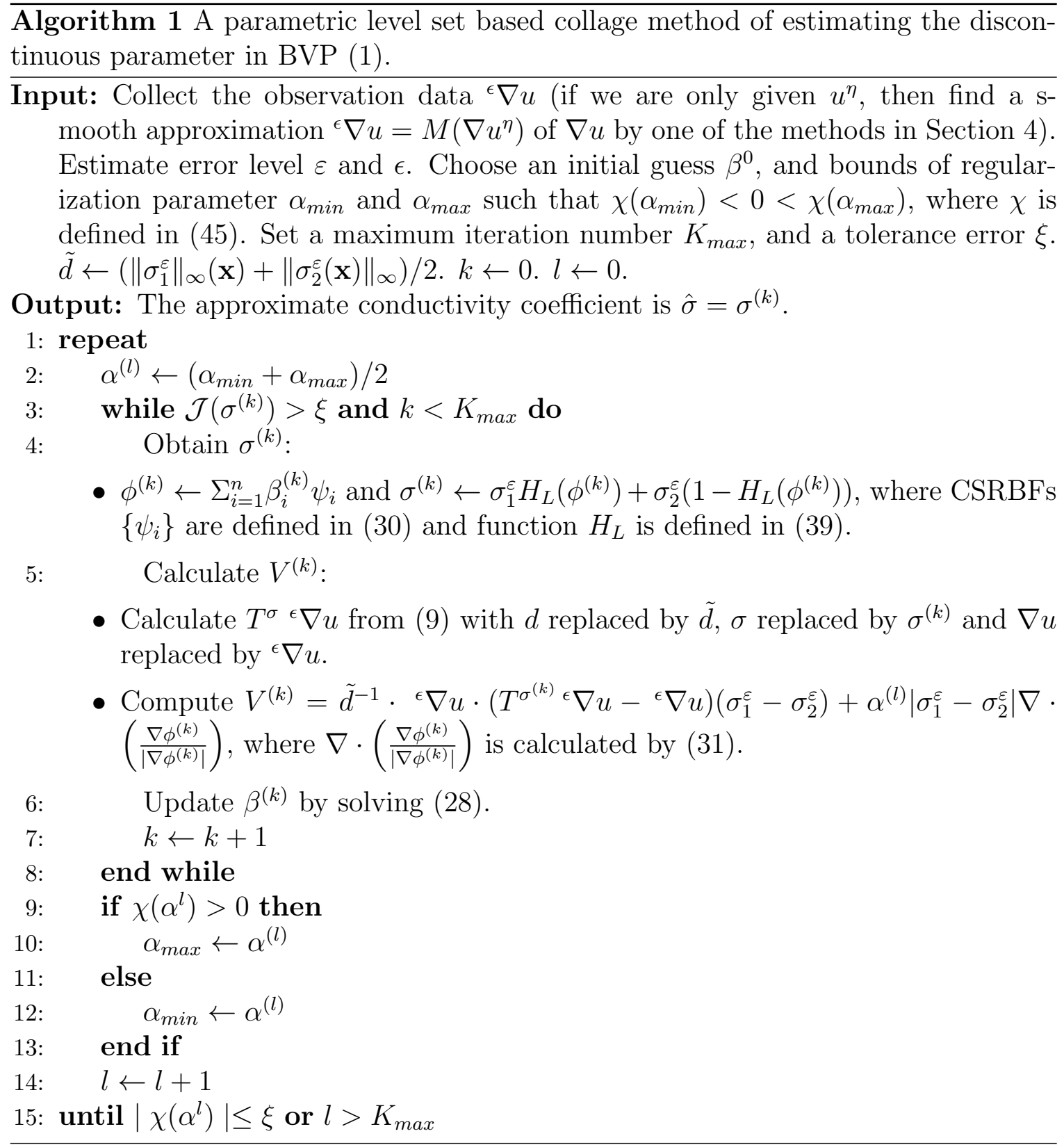

\section{Computer simulations}

To verify the feasibility of our method, we do some numerical experiments in this section. In our numerical experiments, the domain $\Omega=(0,1)^{2}$ is firstly divided into a rectangular mesh with uniform mesh size $h=0.0125$ for both the $x$ and the $y$ variables. The finite element mesh is produced by dividing each rectangle into two triangular elements using the diagonal of positive slope. We use piecewise continuous linear basis functions for the finite element space. The centers $\mathbf{x}=(x, y)$ of the CSRBFs are distributed evenly in $\Omega$ and is generated by the MatLab code $[x, y]=$ meshgrid(0.05: $0.025: 0.95)$. Furthermore, set the support size $s=0.1$.

For better assessing the accuracy of approximate solutions, we define the relative error of the estimated discontinuous coefficient $\hat{\sigma}: \operatorname{Err}(\hat{\sigma})=\|\hat{\sigma}-\bar{\sigma}\|_{L^{2}(\Omega)} /\|\bar{\sigma}\|_{L^{2}(\Omega)}$, where $\bar{\sigma}$ is the true coefficient. 


\subsection{Example 1}

The first numerical experiment is taken from example 2 in [11], i.e., reconstruction a discontinuous coefficient $\sigma$ with a simple geometry; namely, the exact discontinuous coefficient is given by

$$
\sigma= \begin{cases}2 & \text { when } \mathbf{x} \in D \subset \Omega, \\ 1 & \text { when } \mathbf{x} \in \Omega \backslash D\end{cases}
$$

where $D$ is composed by two closed curves, displayed in Fig. 1.

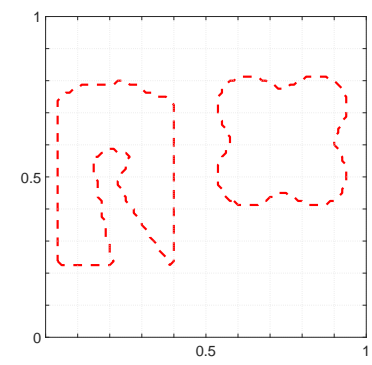

Figure 1: Exact zero level sets.

Let $u=\sin (\pi x) \sin (\pi y)$ be the exact solution. Then, the source function $f$ can be computed by equation (1). Suppose that instead of exact data $\left\{\sigma_{1}(\mathbf{x})=2, \sigma_{2}(\mathbf{x})=\right.$ $1, \nabla u(\mathbf{x})\}$ we are given approximate data $\left\{\sigma_{1}^{\varepsilon}(\mathbf{x}), \sigma_{2}^{\varepsilon}(\mathbf{x}),{ }^{\epsilon} \nabla u(\mathbf{x})\right\}$ such that: $\sigma_{1}^{\varepsilon}(\mathbf{x})=$ $(1+\varepsilon \cdot R d 1(\mathbf{x})) \times 2, \sigma_{2}^{\varepsilon}(\mathbf{x})=(1+\varepsilon \cdot R d 2(\mathbf{x})) \times 1$ and ${ }^{\epsilon} \nabla u(\mathbf{x})=(1+\epsilon \cdot R d 3(\mathbf{x})) \times$ $\nabla u(\mathbf{x})$, where $R d 1(\mathbf{x}), R d 2(\mathbf{x}), R d 3(\mathbf{x})$ are independent and identically distributed (i.i.d.) random variables with an uniform distribution $\mathcal{U}(-1,1)$ and $\varepsilon, \epsilon$ are noise levels.

We first study the case with the small error level $\varepsilon=1 \%$ and $\epsilon=0.1 \%$. The initial guess for $\sigma$ with the location of discontinuities is specified as a circle as shown in (a) of Fig. 2. The evolution of computed zero level set is depicted in (a) - (e) of Fig. 2, while the evolution of the relative error $\operatorname{Err}(\hat{\sigma})$ is shown in (f) of Fig. 2. We see that, starting with one initial curve, our algorithm is able to automatically split it into two pieces and capture the two separate regions successfully. This is the intrinsic advantage of the level set based approaches.

Now, we consider the case with the large error level $\varepsilon=5 \%$ and $\epsilon=1 \%$. The method in [11] fails to identify the zero level set. However, our method can tolerate such noise data, and the approximate shape of the two objects is identified quite well after 400 iterations. The evolutions of computed zero level set and the relative error $\operatorname{Err}(\hat{\sigma})$ are depicted in Fig. 3. Note that in this experiment, we use a different initial curve; see in (a) of Fig. 3. Various numerical experiments demonstrate that the results of our approach are almost independent of the choice of the initial curve. Hence, from now on, we use the same initial curve as shown in (a) of Fig. 3.

In Fig. 4, we show the result of our method for high accuracy data; namely, $\varepsilon=\epsilon=0.1 \%$. Actually, after only 70 steps, the recovered coefficient is already accurate $(\operatorname{Err}(\hat{\sigma})=0.00675$ at 70 th iteration $)$. 


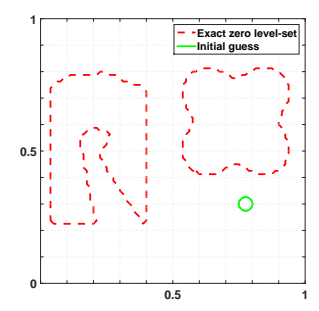

(a)

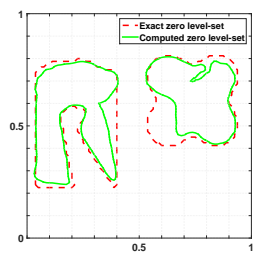

(d)

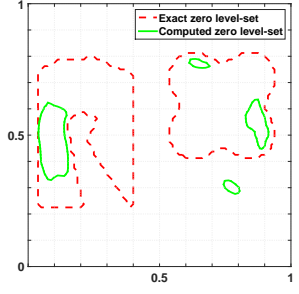

(b)

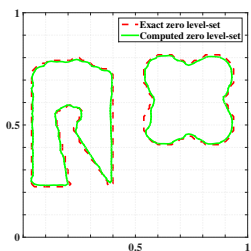

(e)

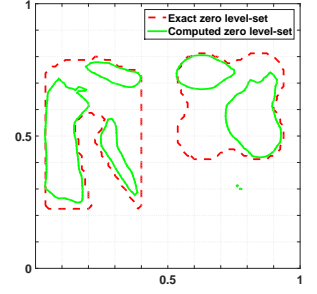

(c)

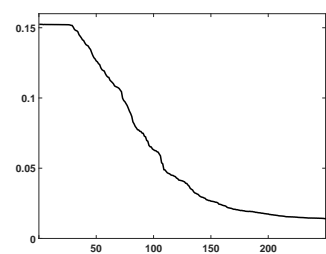

(f)

Figure 2: The identified zero level curve by Algorithm 1 at different iterations with noise level $\varepsilon=1 \%$, $\epsilon=0.1 \%$. (a) Initial guess. $\operatorname{Err}(\hat{\sigma})=0.1524$. (b) After 50 iterations. $\operatorname{Err}(\hat{\sigma})=0.1264$. (c) After 100 iterations. $\operatorname{Err}(\hat{\sigma})=0.0632$. (d) After 150 iterations. $\operatorname{Err}(\hat{\sigma})=0.0266$. (e) After 250 iterations. $\operatorname{Err}(\hat{\sigma})=0.0028$. (f) The evolution of the relative error $\operatorname{Err}(\hat{\sigma})$.



(a)

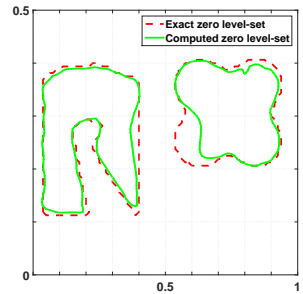

(d)

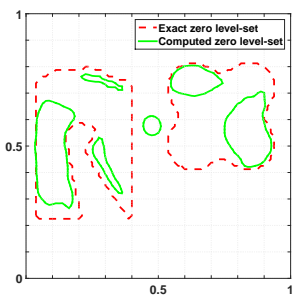

(b)

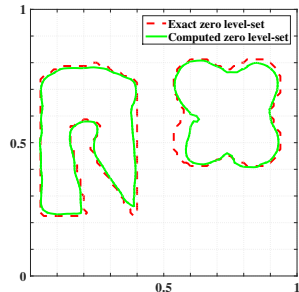

(e)

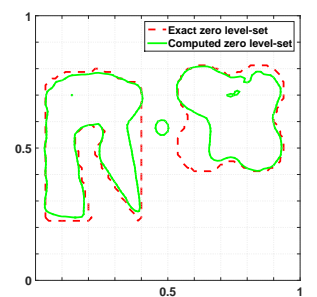

(c)

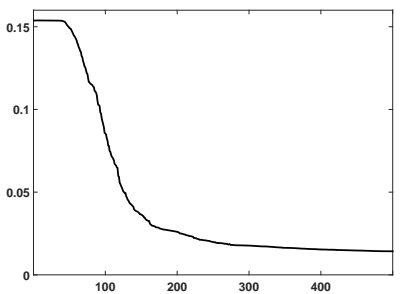

(f)

Figure 3: The identified zero level curve by Algorithm 1 at different iterations with noise level $\varepsilon=5 \%$, $\epsilon=1 \%$. (a) Initial guess. $\operatorname{Err}(\hat{\sigma})=0.1538$. (b) After 100 iterations. $\operatorname{Err}(\hat{\sigma})=0.0855$. (c) After 200 iterations. $\operatorname{Err}(\hat{\sigma})=0.0260$. (d) After 300 iterations. $\operatorname{Err}(\hat{\sigma})=0.0177$. (e) After 400 iterations. $\operatorname{Err}(\hat{\sigma})=0.0153$. (f) The evolution of the relative error $\operatorname{Err}(\hat{\sigma})$.

\subsubsection{Comparison with other state-of-the-art methods}

In order to demonstrate the advantage of our algorithm over traditional approaches, we solve the same problem by two modern PDE-based techniques - a gradient descent method (Algorithm 2) for the optimization problem (6), and an augmented Lagrangian method (Algorithm 3) for the optimization problem (5). See the Appen- 


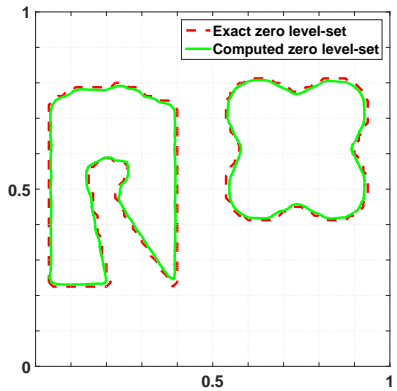

(a)

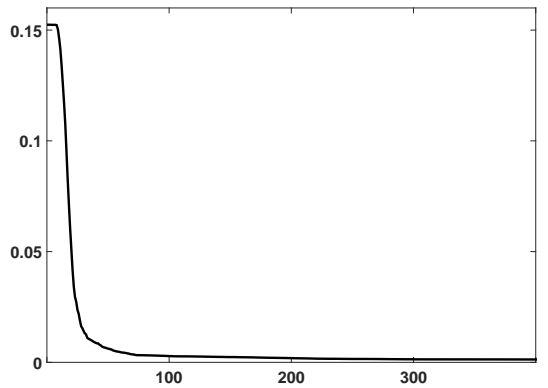

(b)

Figure 4: The identified zero level curve by Algorithm 1 at different iterations with noise level $\varepsilon=0.1 \%, \epsilon=0.1 \%$. (a) the result after 300 iterations. $\operatorname{Err}(\hat{\sigma})=0.0014$. (b) The evolution of the relative error $\operatorname{Err}(\hat{\sigma})$.

dices $\mathrm{B}$ and $\mathrm{C}$ for the details.

To the best of our knowledge, there is no available regularization parameter selection method in the literature for both the gradient descent methods of solving (6) and augmented Lagrangian methods of solving (5). Here, we choose an appropriate value for the regularization parameter after testing Algorithms 2 and 3 with different choices of regularization parameter.

As one can see in figures 5 and 6 , with an appropriate choice of regularization parameter and small noise level, both Algorithms 2 and 3 work as well as our approach. However, with $(5 \%, 1 \%)$ of noise, the identified zero level set by Algorithm 2 is rather poor. Finally, the computational accuracy determinations of the discontinuous coefficient estimates by the collage method (Algorithm 1), the (6) based gradient descent method (Algorithm 2), and the augmented Lagrangian method (Algorithm 3) can be found in Tab. 1. As one can see, with the appropriate choice of the regularization parameter, all of the methods are stable: the more accurate the observation data, the smaller the relative error of the estimated conduction coefficient. However, for the large noise-level data, only our method and Algorithm 3 provide acceptable results. Moreover, Tab. 1 shows that for this model problem, our method is slightly better than Algorithm 3.

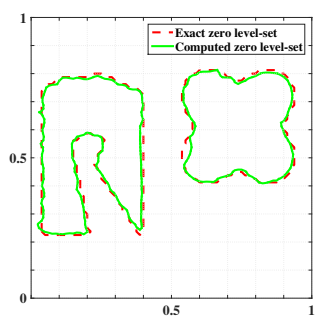

(a)

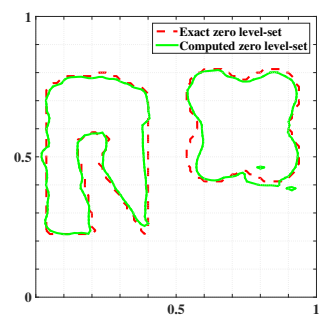

(b)

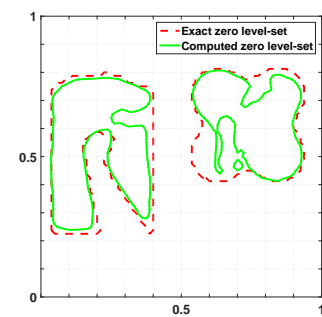

(c)

Figure 5: The identified zero level curve by Algorithm 2 with the appropriate choice of regularization parameter $\alpha$. (a) Noise level: $\varepsilon=\epsilon=0.1 \%$. $\alpha=0.001$. $\operatorname{Err}(\hat{\sigma})=0.0025$. (b) Noise level: $\varepsilon=1 \%$, $\epsilon=0.1 \%$. $\alpha=0.01$. $\operatorname{Err}(\hat{\sigma})=0.0053$. (c) Noise level: $\varepsilon=5 \%, \epsilon=1 \% . \alpha=0.3$. $\operatorname{Err}(\hat{\sigma})=0.0252$. 


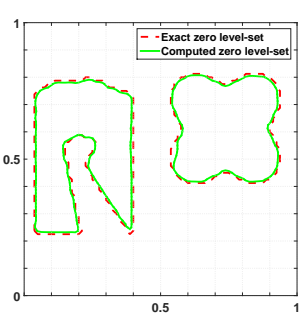

(a)

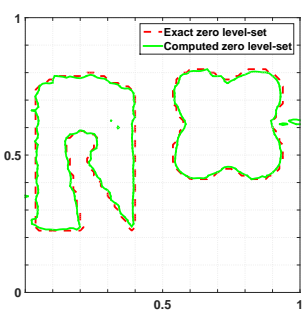

(b)

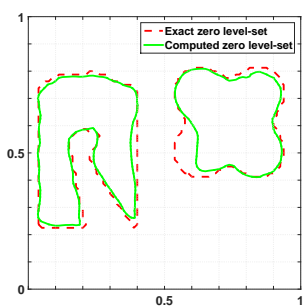

(c)

Figure 6: The identified zero level curve by Algorithm 3 with the appropriate choice of regularization parameter $\alpha$. (a) Noise level: $\varepsilon=\epsilon=0.1 \% . \alpha=0.001 . \operatorname{Err}(\hat{\sigma})=0.0017$. (b) Noise level: $\varepsilon=1 \%$, $\epsilon=0.1 \% . \alpha=0.01 . \operatorname{Err}(\hat{\sigma})=0.0032$. (c) Noise level: $\varepsilon=5 \%, \epsilon=1 \% . \alpha=0.3 . \operatorname{Err}(\hat{\sigma})=0.0172$.

Table 1: The comparison (the relative error $\operatorname{Err}(\hat{\sigma}))$ between the parametric level set based collage method (Algorithm 1), the (6) based gradient descent method (Algorithm 2), and the augmented Lagrangian method (Algorithm 3).

\begin{tabular}{lccc}
\hline Noise level $(\varepsilon, \epsilon)$ & Algorithm 1 & Algorithm 2 & Algorithm 3 \\
\hline$(0.01 \%, 0.01 \%)$ & 0.0014 & 0.0025 & 0.0017 \\
$(0.05 \%, 0.01 \%)$ & 0.0015 & 0.0029 & 0.0019 \\
$(0.1 \%, 0.01 \%)$ & 0.0019 & 0.0031 & 0.0021 \\
$(0.1 \%, 0.05 \%)$ & 0.0020 & 0.0039 & 0.0023 \\
$(0.1 \%, 0.1 \%)$ & 0.0022 & 0.0043 & 0.0023 \\
$(0.5 \%, 0.1 \%)$ & 0.0027 & 0.0044 & 0.0027 \\
$(1 \%, 0.1 \%)$ & 0.0028 & 0.0053 & 0.0032 \\
$(1 \%, 0.5 \%)$ & 0.0030 & 0.0088 & 0.0040 \\
$(1 \%, 1 \%)$ & 0.0045 & 0.0107 & 0.0093 \\
$(5 \%, 0.1 \%)$ & 0.0091 & 0.0153 & 0.0103 \\
$(5 \%, 0.5 \%)$ & 0.0103 & 0.0174 & 0.0107 \\
$(5 \%, 1 \%)$ & 0.0153 & 0.0252 & 0.0172 \\
$(5 \%, 5 \%)$ & 0.0207 & 0.0542 & 0.0324 \\
$(10 \%, 5 \%)$ & 0.0721 & 0.1027 & 0.0994 \\
\hline \hline
\end{tabular}

\subsection{Example 2}

Our second group of experiments deal with some complex geometry. The first experiment is to find the wreck of the crashed aircraft or/and the capsized ship in the ocean; see the left side in Fig. 7 . At room temperature, the heat conductivities of sea water and iron are 0.58 and $80 \mathrm{~W} /(\mathrm{m} \mathrm{K})$, i.e.

$$
\bar{\sigma}(\mathbf{x})= \begin{cases}\bar{\sigma}_{1}(\mathbf{x})=80, & (x, y) \subset D \\ \bar{\sigma}_{2}(\mathbf{x})=0.58, & (x, y) \subset \Omega \backslash D\end{cases}
$$

where domain $D$ describes the location of the seeking aircraft or/and ship. Domain $\Omega \backslash D$ means the sea environment. The exact conductivity representation of the original 


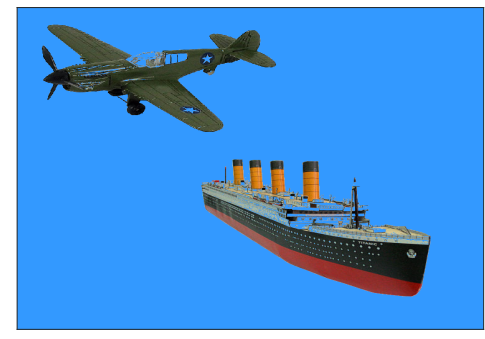

(a) The original picture

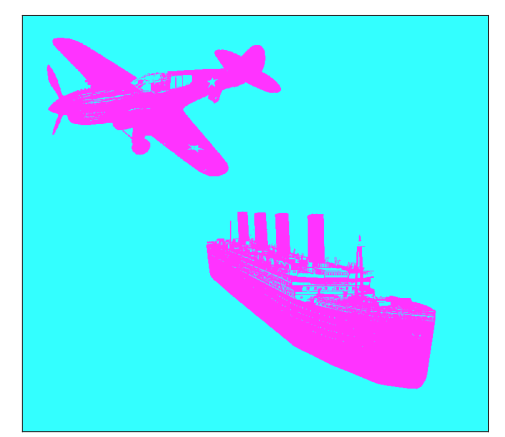

(b) The conductivity representation

Figure 7: The original picture of an aircraft and a ship in the ocean and their conductivity representation.

picture is displayed in the right-hand side of Fig. 7. Let $u=\sin (\pi x) \sin (\pi y)$ be the exact solution. Suppose that the noisy data $\left\{\sigma_{1}^{\varepsilon}(\mathbf{x}), \sigma_{2}^{\varepsilon}(\mathbf{x}), u^{\eta}(\mathbf{x})\right\}$ are generated by the same method as in the previous example, but with the noise levels $(\varepsilon, \eta)=(5 \%, 5 \%)$. Moreover, the effective gradient data ${ }^{\epsilon} \nabla u=M\left(u^{\eta}\right)$ is calculated by the analytic averaging technique in Section 4.2. The process of our algorithm is displayed in Fig. 8, and the evolution of relative errors $\operatorname{Err}(\hat{\sigma})$ is depicted in Fig. 9. As one can see, after 300 steps, the recovered boundary of $D$ is already very accurate.

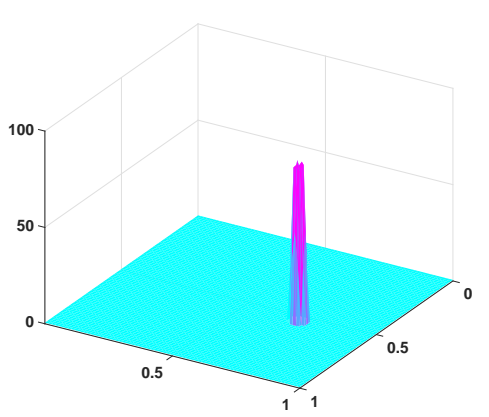

(a) The initial guess

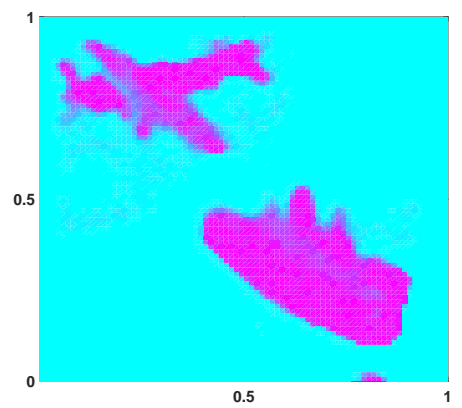

(c) After 300 iterations

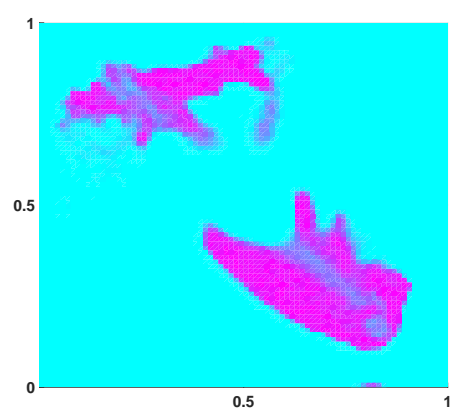

(b) After 100 iterations

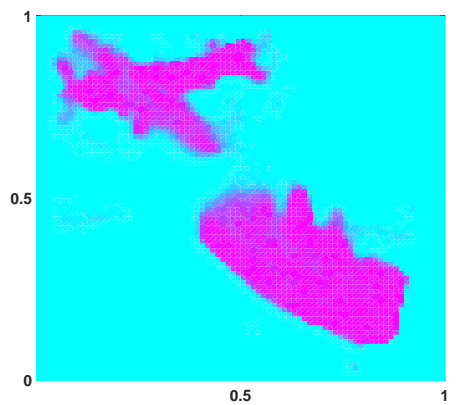

(d) After 900 iterations

Figure 8: The recovered results by Algorithm 1 at different iterations with noise level $\varepsilon=5 \%$, $\eta=5 \%$. 


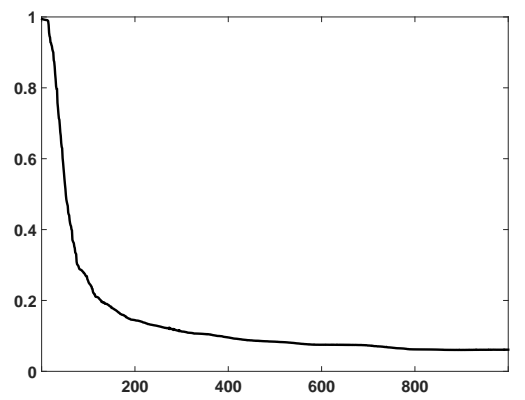

Figure 9: The evolution of the relative error.

In Fig. 10, the reconstruction results corresponding to the different noise levels are displayed. Subfigure (a) in Fig. 10 for $\varepsilon=20 \%$ and $\eta=5 \%$ and Subfigure (b) for $\varepsilon=5 \%$ and $\eta=10 \%$. As one can see, $\sigma(\mathbf{x})$ can be recovered even with large noise levels for this model problem.

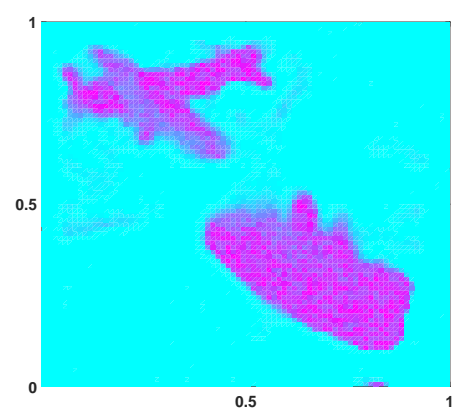

(a) $\varepsilon=20 \%$ and $\eta=5 \%$.

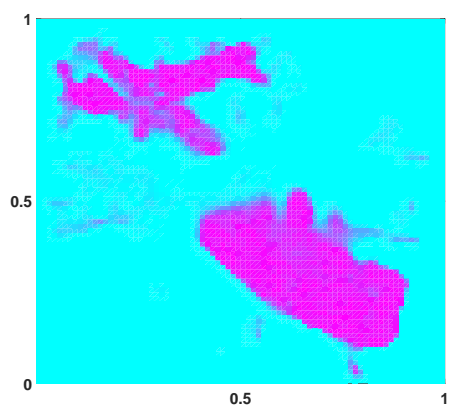

(b) $\varepsilon=5 \%$ and $\eta=10 \%$.

Figure 10: Reconstruction results with respect to different noise levels.

\subsection{Example 3}

In this example, we consider the case with different data structure when a smooth source function is given since, from a practical viewpoint, the source term $f$ does not contain any information about the discontinuity of conduction coefficient $\sigma$. In order to highlight the influence of data structure, we solve Example 1 in Section 7.1 with the solution data set $u$ and the smooth source function $f=\sin (\pi x) \sin (\pi y)$. The same method is used to generate the noisy data $\left\{\sigma_{1}^{\varepsilon}(\mathbf{x}), \sigma_{2}^{\varepsilon}(\mathbf{x}), u^{\eta}(\mathbf{x})\right\}$. The effective value of ${ }^{\epsilon} \nabla u(\mathbf{x})$ is computed through Approach I in Section 4.1, and the same initial guess is used. The results with different noise levels are displayed in Fig. 11. As we can see, similar accurate results can be obtained as in Section 7.1 though we need more iterations. For small noise level, see (a) and (b) in Fig. 11, almost double iterations are required in order to obtain the high accurate reconstructed conduction coefficient. In the case of relative large noise level, see (c) and (d) in Fig. 11, only slight more iterations are needed for obtaining the similar accuracy of the estimated conduction coefficient $\hat{\sigma}$. 


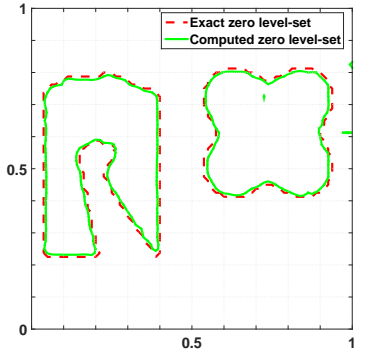

(a)

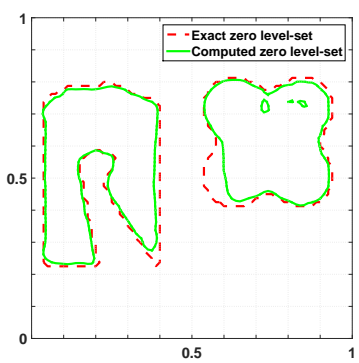

(c)

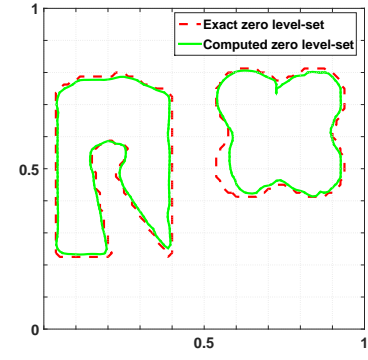

(b)

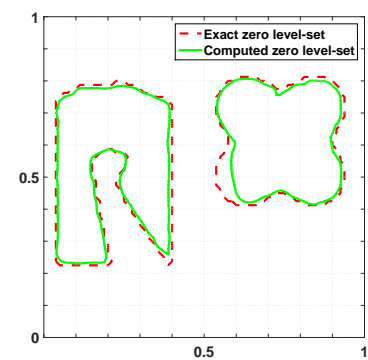

(d)

Figure 11: The identified zero level curve by Algorithm 1 with data $u$ and smooth source function $f=\sin (\pi x) \sin (\pi y)$. (a) Noise level: $\varepsilon=\eta=0.1 \%$. Iteration number: 500 . $\operatorname{Err}(\hat{\sigma})=0.0024$. (b) Noise level: $\varepsilon=1 \%, \eta=0.1 \%$. Iteration number: 600. $\operatorname{Err}(\hat{\sigma})=0.0081$. (c) Noise level: $\varepsilon=5 \%$, $\eta=1 \%$. Iteration number: 400. $\operatorname{Err}(\hat{\sigma})=0.0127$. (d) Noise level: $\varepsilon=5 \%, \eta=1 \%$. Iteration number: 600. $\operatorname{Err}(\hat{\sigma})=0.0093$.

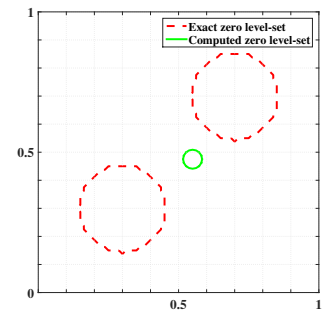

(a) Initial guess

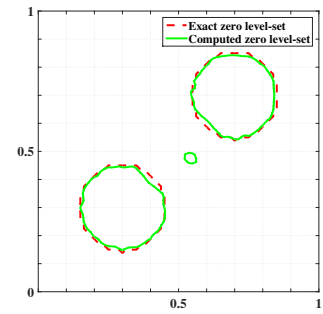

(d) 100 iterations

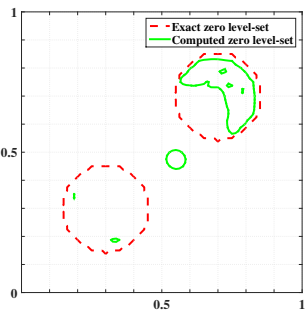

(b) 20 iterations

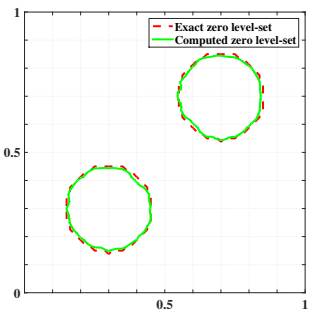

(e) 180 iterations

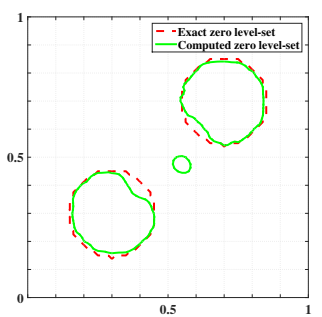

(c) 50 iterations

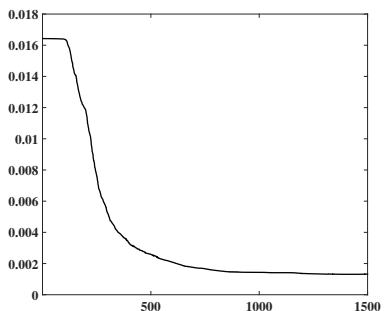

(f) The evolution of the relative error $\operatorname{Err}(\hat{\sigma})$

Figure 12: The computed zero level sets by Algorithm 1 at different iterations with noise level $\varepsilon=\eta=1 \%$ and the evolution of the relative error $\operatorname{Err}(\hat{\sigma})$. 


\subsection{Example 4}

In the last example, we consider the case when the exact discontinuous coefficient is not a piecewise constant. Let

$$
\bar{\sigma}=\left\{\begin{aligned}
0.35+0.1 \exp (-x-y) & \text { when } \mathbf{x} \in D \subset \Omega \\
0.58 & \text { when } \mathbf{x} \in \Omega \backslash D
\end{aligned}\right.
$$

where $D$ is composed by two circles with radius 0.15 whose centers are $(0.7,0.7)$ and $(0.3,0.3)$ respectively. Let $\hat{u}$ be the exact finite element solution for the exact source function $f=\sin (\pi x) \sin (\pi y)$ and coefficient $\bar{\sigma}$. Suppose that the approximate data $\left\{\sigma_{1}^{\varepsilon}(\mathbf{x}), \sigma_{2}^{\varepsilon}(\mathbf{x}), u^{\eta}(\mathbf{x})\right\}$ are defined through the same method as before, with noise levels $(\varepsilon, \eta)=(1 \%, 1 \%)$ and $(\varepsilon, \eta)=(5 \%, 5 \%)$ respectively. Moreover, the effective gradient data ${ }^{\epsilon} \nabla u=M\left(u^{\eta}\right)$ is calculated by the smoothing approach introduced in Section 4.1. The evolution of the computed zero level set are depicted in (a) - (e) of figures 12 and 13 respectively, while the evolution of the relative errors $\operatorname{Err}(\hat{\sigma})$ are shown in (f) of figures 12 and 13 respectively.

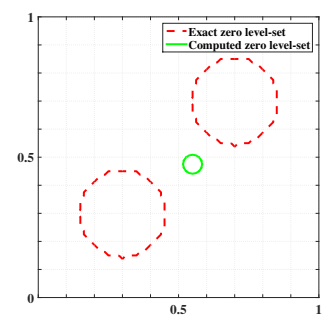

(a) Initial guess



(d) 500 iterations

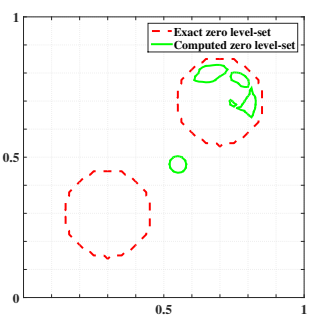

(b) 150 iterations

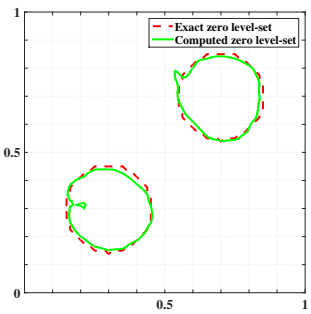

(e) 1500 iterations

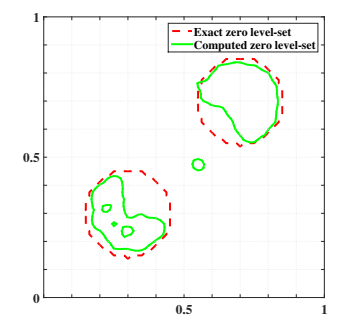

(c) 300 iterations

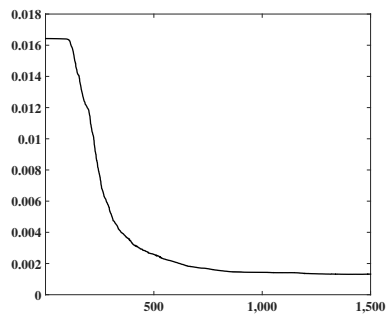

(f) The evolution of the relative error $\operatorname{Err}(\hat{\sigma})$

Figure 13: The computed zero level sets by Algorithm 1 at different iterations with noise level $\varepsilon=\eta=5 \%$ and the evolution of the relative error $\operatorname{Err}(\hat{\sigma})$.

\section{Conclusion}

The numerical experiments performed above indicate that the parametric level set-based collage method is an efficient approach to identify the discontinuous conductivity coefficients in elliptic PDEs. In particular, this method avoids solving adjoint equations when applying the gradient descent method to solve the corresponding optimization problem. Moreover, compared to the traditional level set method, which has to know the value of $\phi(\mathbf{x})$ at every point in the domain $\Omega$, applying the parametric 
level set method, we only need to recover the finite parameters in (25), which greatly reduces the number of the unknowns. The application of this work is of great help in medical image, geophysics and water resource problems.

\section{Acknowledgement}

The work of the first two authors are partially supported by NSCF (No. 91130004) and STINT (No. IB2015-5989). The work of the last author is supported by the Alexander von Humboldt foundation through a postdoctoral researcher fellowship.

\section{Reference}

[1] W. Daily, A. L. Ramirez, Electrical imaging of engineered hydraulic barriers, Geophysics 65 (1) (2000) 83-94.

[2] K. Dines, R. J. Lytle, Analysis of electrical conductivity imaging, Geophysics 46 (7) (1981) 1025-1036.

[3] D. C. Dobson, F. Santosa, An image-enhancement technique for electrical impedance tomography, Inverse Probl. 10 (2) (1994) 317.

[4] R. E. Ewing, T. Lin, A class of parameter estimation techniques for fluid flow in porous media, Adv. Water Resour. 14 (2) (1991) 89-97.

[5] T. F. Chan, X. C. Tai, Identification of discontinuous coefficients in elliptic problems using total variation regularization, SIAM J. Sci. Comput. 25 (3) (2003) 881-904.

[6] Z. M. Chen, J. Zou, An augmented lagrangian method for identifying discontinuous parameters in elliptic systems, SIAM J. Control Optim. 37 (3) (1999) 892-910.

[7] O. Dorn, E. L. Miller, C. M. Rappaport, A shape reconstruction method for electromagnetic tomography using adjoint fields and level sets, Inverse Probl. 16 (5) (2000) 1119-1156.

[8] O. Dorn, D. Lesselier, Level set methods for inverse scattering, Inverse Probl. 22 (4) (2006) R67-R131.

[9] O. Dorn, D. Lesselier, Level set methods for inverse scattering-some recent developments, Inverse Probl. 25 (12).

[10] K. Ito, K. Kunisch, Z. Li, Level-set function approach to an inverse interface problem, Inverse Probl. 17 (5) (2001) 1225-1242.

[11] T. F. Chan, X. C. Tai, Level set and total variation regularization for elliptic inverse problems with discontinuous coefficients, J. Comput. Phys. 193 (1) (2004) $40-66$.

[12] X. C. Tai, H. Li, A piecewise constant level set method for elliptic inverse problems, Appl. Numer. Math. 57 (5-7) (2007) 686-696. 
[13] L. K. Nielsen, X. C. Tai, S. I. Aanonsen, M. Espedal, A binary level set model for elliptic inverse problems with discontinuous coefficients, Int. J. Numer. Anal. Model. 4 (1) (2007) 74-99.

[14] T. Gu, H. Li, L. Zhang, L. Gao, A level set method for structural shape and topology optimization using radial basis function, in: Computer Supported Cooperative Work in Design (CSCWD), Proceedings of the 2014 IEEE 18th International Conference on, IEEE, 2014, pp. 408-413.

[15] Z. Luo, L. Y. Tong, Z. Kang, A level set method for structural shape and topology optimization using radial basis functions, Comput. Struct. 87 (7-8) (2009) 425434.

[16] C. A. Zhuang, Z. H. Xiong, H. Ding, Topology optimization of multi-material for the heat conduction problem based on the level set method, Eng. Optimiz. 42 (9) (2010) 811-831.

[17] G. Pingen, M. Waidmann, A. Evgrafov, K. Maute, A parametric level-set approach for topology optimization of flow domains, Struct. Multidiscip. Optim. 41 (1) (2010) 117-131.

[18] S. Y. Wang, M. Y. Wang, Radial basis functions and level set method for structural topology optimization, Int. J. Numer. Methods Eng. 65 (12) (2006) 20602090 .

[19] A. Aghasi, M. Kilmer, E. L. Miller, Parametric level set methods for inverse problems, SIAM J. Imaging Sci. 4 (2) (2011) 618-650.

[20] B. Michael, Fractals Everywhere, Academic Press, New York, 1988.

[21] H. E. Kunze, J. E. Hicken, E. R. Vrscay, Inverse problems for odes using contraction maps and suboptimality of the 'collage method', Inverse Probl. 20 (3) (2004) 977-991.

[22] V. Capasso, H. E. Kunze, D. La Torre, E. R. Vrscay, Solving inverse problems for biological models using the collage method for differential equations, J. Math. Biol. 67 (1) (2013) 25-38.

[23] H. E. Kunze, D. La Torre, E. R. Vrscay, Random fixed point equations and inverse problems using "collage method" for contraction mappings, J. Math. Anal. Appl. 334 (2) (2007) 1116-1129.

[24] K. M. Levere, H. Kunze, Using the collage method to solve one-dimensional twopoint boundary value problems at steady-state, Nonlinear Anal.-Theory Methods Appl. 71 (12) (2009) e1487-e1495.

[25] W. P. Ziemer, Weakly differentiable functions, Spriger-Verlag, New York.

[26] M. D. Buhmann, Radial basis functions, Acta Numer. 9 (2000) 1-38. 
[27] A. Gelas, O. Bernard, D. Friboulet, R. Prost, Compactly supported radial basis functions based collocation method for level-set evolution in image segmentation, IEEE Trans. Image Process. 16 (7) (2007) 1873-1887.

[28] M. Hanke, O. Scherzer, Inverse problems light: numerical differentiation, The American Mathematical Monthly 108 (6) (2001) 512-521.

[29] B. Engquist, Y. H. Tsai, Heterogeneous multiscale methods for stiff ordinary differential equations, Math. Comput. 74 (252) (2005) 1707-1742.

[30] M. Gulliksson, A. Holmbom, J. Persson, Y. Zhang, A separating oscillation method of recovering the g-limit in standard and non-standard homogenization problems, Inverse Problems 32 (2) (2016) 025005.

[31] A. N. Tikhonov, A. S. Leonov, A. G. Yagola, Nonlinear ill-posed problems, Chapman and Hall, London, 1998.

[32] A. S. Leonov, On the total-variation convergence of regularizing algorithms for ill-posed problems, Comput. Math. Math. Phys. 47 (5) (2007) 732-747.

[33] H. W. Engl, M. Hanke, A. Neubauer, Regularization of inverse problems, Vol. 375, Springer Science \& Business Media, 1996.

[34] C. R. Vogel, Computational methods for inverse problems, Vol. 23, SIAM, 2002.

[35] A. V. Goncharsky, A. S. Leonov, A. G. Yagola, A generalized residual principle, Zh.Vychisl.mat.mat.fiz. 13 (2) (1973) 294-302.

\section{Appendix A. Proof of Theorem 9.}

1). Without loss of generality, here we only show the continuity of operator $A$. Fix a point $\sigma_{0} \in \Theta$, then $A \sigma_{0} \in L^{2}(\Omega)^{N}$ satisfies

$$
\int_{\Omega} \tilde{d} \cdot A \sigma_{0} \cdot \nabla v d \mathbf{x}=\int_{\Omega} f v+\left(\tilde{d}-\sigma_{0}\right) \nabla u \cdot \nabla v d \mathbf{x}, \quad \forall v \in H_{0}^{1}(\Omega) .
$$

Hence, $\forall \sigma \in \Theta$, we have

$$
\int_{\Omega} \tilde{d} \cdot\left(A \sigma-A \sigma_{0}\right) \cdot \nabla v d \mathbf{x}=\int_{\Omega}\left(\sigma_{0}-\sigma\right) \nabla u \cdot \nabla v d \mathbf{x}, \quad \forall v \in H_{0}^{1}(\Omega),
$$

Owing that $\tilde{d}$ is constant function, and substitute $v$ by $A \sigma-A \sigma_{0}$, it's easy to obtain

$$
\left\|A \sigma-A \sigma_{0}\right\|_{L^{2}(\Omega)^{N}} \leq \tilde{d}^{-1} \cdot\left\|\sigma-\sigma_{0}\right\|_{\infty} \cdot\|\nabla u\|_{L^{2}(\Omega)^{N}},
$$

which implies the continuity of operator $A$ on $L^{\infty}(\Omega)$.

2 ). By definitions of operator $A$ and $A_{\epsilon}$ (in (36) and (38)), $\forall v \in H_{0}^{1}(\Omega)$

$$
\int_{\Omega} \tilde{d} \cdot A \sigma \cdot \nabla v d \mathbf{x}=\int_{\Omega} f v+(\tilde{d}-\sigma) \cdot \nabla u \cdot \nabla v d \mathbf{x}
$$


and

$$
\int_{\Omega} \tilde{d} \cdot A_{\epsilon} \sigma \cdot \nabla v d \mathbf{x}=\int_{\Omega} f v+(\tilde{d}-\sigma) \cdot{ }^{\epsilon} \nabla u \cdot \nabla v d \mathbf{x}
$$

Subtracting above two equation we obtain

$$
\int_{\Omega} \tilde{d} \cdot\left(A_{\epsilon} \sigma-A \sigma\right) \cdot \nabla v d \mathbf{x}=\int_{\Omega}(\tilde{d}-\sigma)\left({ }^{\epsilon} \nabla u-\nabla u\right) \cdot \nabla v d \mathbf{x}, \quad \forall v \in H_{0}^{1}(\Omega) .
$$

Replace $\nabla v$ by $\left(A_{\epsilon} \sigma-A \sigma\right)$ and use the Cauchy-Schwarz inequality, $\forall \sigma \in \Theta$ or $\widehat{\Theta}_{\varepsilon}$, we get

$$
\begin{aligned}
\left\|A_{\epsilon} \sigma-A \sigma\right\|_{L^{2}(\Omega)^{N}} & \leq\left\|\frac{\tilde{d}-\sigma}{\tilde{d}}\right\|_{L^{\infty}(\Omega)} \cdot\left\|^{\epsilon} \nabla u-\nabla u\right\|_{L^{2}(\Omega)^{N}} \\
& =\sqrt{\lambda(\tilde{d}, \sigma)} \cdot \epsilon<\epsilon \leq(1+\Psi(\sigma)) \epsilon,
\end{aligned}
$$

which implies our assertion with $\Upsilon(s, t) \equiv(1+t) s$.

$3.1)$. Define $\widehat{\Psi}(\sigma):=\sup _{v \in V} \int_{\Omega}(-\sigma \cdot \nabla \cdot v) d \mathbf{x}$, where the set of test functions $V=$ $\left\{v \in C_{0}^{1}(\Omega)^{2}: \forall \mathbf{x} \in \Omega,|v(\mathbf{x})| \leq 1\right\}$. Here, $|v(\mathbf{x})|=\sqrt{v_{1}^{2}(\mathbf{x})+v_{2}^{2}(\mathbf{x})}$. By [34] we have $\widehat{\Psi}(\sigma)=\Psi(\sigma)$ for $\sigma$ in the Sobolev space $W^{1,1}(\Omega)$. Similarly, it is not difficult to show that $\widehat{\Psi}(\sigma)=\Psi(\sigma)$ for $\sigma \in \widehat{\Theta}_{\varepsilon}$. Hence, we only need to prove the lower semicontinuity of the functional $\widehat{\Psi}$. Note that the strongly convergence in $L^{\infty}(\Omega)$ implies the weak convergence in $L^{1}(\Omega)$. Suppose that $\sigma_{n} \rightarrow \bar{\sigma}$. Then, for any fixed $v \in V$ we have

$$
\begin{array}{r}
\int_{\Omega}(-\bar{\sigma} \cdot \nabla \cdot v) d \mathbf{x}=\lim _{n \rightarrow \infty} \int_{\Omega}\left(-\sigma_{n} \cdot \nabla \cdot v\right) d \mathbf{x}=\liminf _{n \rightarrow \infty} \int_{\Omega}\left(-\sigma_{n} \cdot \nabla \cdot v\right) d \mathbf{x} \\
\leq \liminf _{n \rightarrow \infty} \sup _{\zeta \in V} \int_{\Omega}\left(-\sigma_{n} \cdot \nabla \cdot \zeta\right) d \mathbf{x}=\liminf _{n \rightarrow \infty} \widehat{\Psi}\left(\sigma_{n}\right) .
\end{array}
$$

Taking the supremum over $v \in V$ gives $\widehat{\Psi}(\bar{\sigma}) \leq \liminf _{n \rightarrow \infty} \widehat{\Psi}\left(\sigma_{n}\right)$, which yields the required results since $\widehat{\Psi}(\bar{\sigma})=\Psi(\bar{\sigma})$ and $\widehat{\Psi}\left(\sigma_{n}\right)=\Psi\left(\sigma_{n}\right)$ for $\bar{\sigma}, \sigma_{n} \in \widehat{\Theta}_{\varepsilon}$.

3.2). First, by the definition of $\sigma$ in (39) it is easy to show that $\sigma$ is a function with bounded Lipschitz constant. Then, by the Arzela-Ascoli theorem, which says that a uniformly bounded sequence of functions with bounded Lipschitz constant has a convergent subsequence, we obtain that $\Xi:=\left\{\sigma \in \widehat{\Theta}_{\varepsilon}:\|\sigma\|_{L^{\infty}(\Omega)} \leq\left\|\sigma_{1}^{\varepsilon}\right\|_{L^{\infty}(\Omega)}+\right.$ $\left.\left\|\sigma_{2}^{\varepsilon}\right\|_{L^{\infty}(\Omega)}+2 \varepsilon\right\}$ is a compact set in $L^{\infty}(\Omega)$. Furthermore, by relation $\Psi_{C} \subset \Xi$ and the fact that all closed subsets of compact sets are compact we proved the assertion.

3.3). By Lemma 4, the inequality holds obviously for $\sigma \in \Theta$. Similarity, it is easy to prove the same inequality for $\sigma \in \widehat{\Theta}_{\varepsilon}$.

4). Fist, it is easy to show that $\lim _{\alpha \rightarrow 0} \chi(\alpha) \leq-\omega(\varepsilon, \epsilon)<0$. Furthermore, note that, for sufficiently large $\alpha$, we have $\sigma_{\varepsilon, \epsilon}^{\alpha(\varepsilon, \epsilon)} \in\left\{\sigma_{1}^{\varepsilon}, \sigma_{2}^{\varepsilon}\right\}$, thus, under condition $\varpi>\omega(\varepsilon, \epsilon)$, it is not difficult to show that $\lim _{\alpha \rightarrow \infty} \chi(\alpha) \geq \varpi-\omega(\varepsilon, \epsilon)>0$, which implies that the generalized discrepancy function (45) has a positive solution. Finally, by the standard argument in regularization theory [31, 35], this positive solution guarantees the regularity conditions (41) and (42), and we proved the theorem completely. 


\section{Appendix B. A gradient descent method for problem (6).}

Lemma 10. The derivative of objective functional $\widetilde{\mathcal{J}}$ in optimization problem (6) is

$$
\frac{\partial \widetilde{\mathcal{J}}}{\partial \sigma}=-\nabla u(\sigma) \cdot\left(\nabla u(\sigma)-{ }^{\epsilon} \nabla u\right)+\alpha \frac{\partial \Psi(\sigma)}{\partial \sigma},
$$

where $u(\sigma)$ is the solution of BVP (1) with the fixed parameter $\sigma$.

ProOF. The gradient of $\widetilde{\mathcal{J}}$ with respect to $\sigma$ in the direction $\delta \sigma$ is

$$
\begin{aligned}
\frac{\partial \widetilde{\mathcal{J}}}{\partial \sigma}[\delta \sigma] & =\left\langle\sigma \cdot \frac{\partial \nabla u(\sigma)}{\partial \sigma}[\delta \sigma], \nabla u(\sigma)-{ }^{\epsilon} \nabla u\right\rangle_{L^{2}(\Omega)^{N}}+\alpha \frac{\partial \Psi(\sigma)}{\partial \sigma}[\delta \sigma] \\
& =\left\langle\sigma \cdot \widetilde{M}, \nabla u(\sigma)-{ }^{\epsilon} \nabla u\right\rangle_{L^{2}(\Omega)^{N}}+\alpha \frac{\partial \Psi(\sigma)}{\partial \sigma}[\delta \sigma],
\end{aligned}
$$

where $\widetilde{M} \in L^{2}(\Omega)^{N}$ satisfies

$$
\int_{\Omega} \sigma \cdot \widetilde{M} \cdot \nabla v d \mathbf{x}=-\int_{\Omega} \delta \sigma \nabla u(\sigma) \cdot \nabla v d \mathbf{x}, \quad \forall v \in H_{0}^{1}(\Omega) .
$$

Let $\nabla v=\nabla u(\sigma)-{ }^{\epsilon} \nabla u$ in the above equation, and we obtain

$$
\frac{\partial \widetilde{\mathcal{J}}}{\partial \sigma}[\delta \sigma]=-\left\langle\delta \sigma, \nabla u(\sigma) \cdot\left(\nabla u(\sigma)-{ }^{\epsilon} \nabla u\right)\right\rangle_{L^{2}(\Omega)^{N}}+\alpha \frac{\partial \Psi(\sigma)}{\partial \sigma}[\delta \sigma] .
$$

Therefore, we have

$$
\frac{\partial \widetilde{\mathcal{J}}}{\partial \sigma}=-\nabla u(\sigma) \cdot\left(\nabla u(\sigma)-{ }^{\epsilon} \nabla u\right)+\alpha \frac{\partial \Psi(\sigma)}{\partial \sigma} .
$$

Using the above lemma, it is not difficult to develop a gradient-based algorithm of solving problem (6); see Algorithm 2 in detail. Note that the formulation (5) cannot be solved by the traditional gradient-based methods, due to the difficult calculation of the derivative of the objective functional in (5).

\section{Algorithm 2 A gradient decent method of solving (6).}

Input: Observation data ${ }^{\epsilon} \nabla u$. Parameters $\left\{\beta^{0}, \alpha, K_{\max }\right\} . k \leftarrow 0$.

Output: The approximate conductivity coefficient is $\hat{\sigma}=\sigma^{(k)}$.

1: while $\widetilde{\mathcal{J}}\left(\sigma^{(k)}\right)>\xi$ and $k<K_{\max }$ do

2: $\quad$ Obtain $\sigma^{(k)}$ :

- $\phi^{(k)} \leftarrow \Sigma_{i=1}^{n} \beta_{i}^{(k)} \psi_{i}$ and $\sigma^{(k)} \leftarrow \sigma_{1}^{\varepsilon} H_{L}\left(\phi^{(k)}\right)+\sigma_{2}^{\varepsilon}\left(1-H_{L}\left(\phi^{(k)}\right)\right)$, where CSRBFs $\left\{\psi_{i}\right\}$ are defined in (30) and function $H_{L}$ is defined in (39).

3: $\quad$ Calculate $V^{(k)}$ :

$$
V^{(k)} \leftarrow \nabla u\left(\sigma^{k}\right) \cdot\left(\nabla u\left(\sigma^{k}\right)-{ }^{\epsilon} \nabla u\right)\left(\sigma_{1}^{\varepsilon}-\sigma_{2}^{\varepsilon}\right)+\alpha\left|\sigma_{1}^{\varepsilon}-\sigma_{2}^{\varepsilon}\right| \nabla \cdot\left(\frac{\nabla \phi^{(k)}}{\left|\nabla \phi^{(k)}\right|}\right) .
$$

4: $\quad$ Update $\beta^{(k)}$ by solving (28).

5: $\quad k \leftarrow k+1$

6: end while 


\section{Appendix C. A augmented Lagrangian method for problem (5).}

Define the equation error $e(\sigma, u)$ as the solution of $(\nabla e, \nabla v)_{L^{2}(\Omega)^{N}}:=(\sigma \nabla u, \nabla v)_{L^{2}(\Omega)^{N-}}$ $(f, v)_{L^{2}(\Omega)}, \forall v \in H_{0}^{1}(\Omega)$. Introduce the augmented Lagrangian functional $\mathcal{L}_{r}$ as

$\mathcal{L}_{r}(\sigma, u, \lambda)=\frac{1}{2}\left\|\nabla u(\sigma)-{ }^{\epsilon} \nabla u\right\|_{L^{2}(\Omega)^{N}}^{2}+\alpha \Psi(\sigma)+\frac{r}{2}\|\nabla e(\sigma, u)\|_{L^{2}(\Omega)^{N}}^{2}+(\nabla \lambda, \nabla e(\sigma, u))_{L^{2}(\Omega)^{N}}$.

In this work, we parameterize $\sigma=: \sigma(\beta)$ by the level set function $\phi(\mathbf{x})$ and the radial basis functions $\Phi(\mathbf{x}): \sigma(\mathbf{x})=\sigma_{1}(\mathbf{x}) H(\phi(\mathbf{x}))+\sigma_{2}(\mathbf{x})(1-H(\phi(\mathbf{x}))), \phi(\mathbf{x})=$ $\sum_{i=1}^{n} \beta_{i} \cdot \psi\left(\left\|\mathbf{x}-\mathbf{x}_{i}\right\|\right)=: \Phi(\mathbf{x}) \cdot \beta$. Then, a augmented Lagrangian method of solving problem (5) can be described by Algorithm 3.



